Encyclopedia of AIDS 
Thomas J. Hope - Douglas D. Richman Mario Stevenson

Editors-in-Chief

\section{Encyclopedia of AIDS}

With 341 Figures and 123 Tables

第 Springer 
Department of Cell and Molecular Biology VA San Diego Healthcare System and

Northwestern University

Robert H. Lurie Medical Research Center La Jolla, CA, USA

Chicago, IL, USA

Mario Stevenson

Department of Medicine

University of Miami Leonard M. Miller

School of Medicine

Miami, FL, USA

ISBN 978-1-4939-7100-8

ISBN 978-1-4939-7101-5 (eBook)

ISBN 978-1-4939-7102-2 (print and electronic bundle)

https://doi.org/10.1007/978-1-4939-7101-5

Library of Congress Control Number: 2017957594

\section{(C) Springer Science+Business Media LLC 2018}

This work is subject to copyright. All rights are reserved by the Publisher, whether the whole or part of the material is concerned, specifically the rights of translation, reprinting, reuse of illustrations, recitation, broadcasting, reproduction on microfilms or in any other physical way, and transmission or information storage and retrieval, electronic adaptation, computer software, or by similar or dissimilar methodology now known or hereafter developed.

The use of general descriptive names, registered names, trademarks, service marks, etc. in this publication does not imply, even in the absence of a specific statement, that such names are exempt from the relevant protective laws and regulations and therefore free for general use.

The publisher, the authors and the editors are safe to assume that the advice and information in this book are believed to be true and accurate at the date of publication. Neither the publisher nor the authors or the editors give a warranty, express or implied, with respect to the material contained herein or for any errors or omissions that may have been made. The publisher remains neutral with regard to jurisdictional claims in published maps and institutional affiliations.

\section{Printed on acid-free paper}

This Springer imprint is published by Springer

The registered company is Springer Science+Business Media, LLC

The registered company address is: 233 Spring Street, New York, NY 10013, U.S.A. 


\section{Preface}

The HIV/AIDS epidemic has spanned the past three-and-a-half decades. During this time, the epidemic has caused suffering on a global scale. In the fight to end this epidemic, researchers, healthcare agencies, and governments have mobilized and, as a result of these efforts, have fundamentally altered the course of the epidemic and transformed the prognosis for millions of individuals living with HIV/AIDS. Research has been central to the effort to control the HIV/AIDS epidemic. Early studies aimed at defining key processes in the viral replication cycle helped promote the identification of antiviral agents that have dramatically altered the outlook for infected individuals. This has created a status quo where HIV-1 infection is no longer a death sentence but a manageable, chronic infection. Now the frontier in the fight against HIV/AIDS has shifted to preventing infection, either through use of antivirals or through development of a vaccine. The field has also embarked on seeking ways to eliminate the virus from infected individuals. The hope is that these endeavors will bring an end to the "Age of AIDS."

The human endeavor around three-and-a-half decades of research on HIV/AIDS has produced a large body of scientific information. There are many excellent treatises that have focused on condensing and highlighting different areas of the HIV/AIDS field. Therefore, why an AIDS Encyclopedia? Do we really need another work to summarize what we know about HIV/AIDS?

The AIDS Encyclopedia represents an AIDS "Wikipedia." It is a dynamic, online reference work that can be updated by researchers and by editors that will be able to keep pace with developments in the field. The ability to continuously update the encyclopedia essentially means that it will retain its relevance as an easily accessible and important source of information for students, researchers, and clinicians whether they be working in academia, industry, or the hospital sectors.

Work on the encyclopedia began in 2010 and has seen fruition, thanks to the efforts of many individuals. The editors would like to thank Ms. Tina Shelton, Ms. Rita Beck, and Ms. Sunali Mull at Springer for much of the groundwork in structuring the project and maintaining timelines. Thank you for your persistence and commitment to bringing the project to completion. We would also like to thank the sub-editors who took on responsibility for the 282 individual chapters. We hope the research community will further help in keeping the AIDS Encyclopedia an important and current reference work for the 
HIV/AIDS field, and we welcome contributions in this regard. Finally, the editors would like to acknowledge individuals living with HIV/AIDS who, by participating in research studies, have been central in the effort to end this epidemic.

Chicago, IL, USA

La Jolla, CA, USA

Miami, FL, USA

August 2017
Thomas J. Hope

Douglas D. Richman

Mario Stevenson 


\section{List of Topics}

\section{Anatomic Compartments}

\section{Section Editor: Babafemi O. Taiwo}

Anatomic Compartments as a Barrier to HIV Cure Antiretroviral Drug Penetration into Lymphoid Tissue

Antiretroviral Drug Penetration into the CNS Compartment

HIV Compartments and Viral Rebound During

Treatment Interruption

HIV Coreceptor Tropism in Different Reservoirs

HIV Reservoirs Within the Lungs

HIV Reservoirs in Lymph Nodes and Spleen

HIV Reservoirs in the Central Nervous System HIV-1 Pathogenesis in the Gut

Mucosal Pathogenesis in SIV Infection

\section{Behavioral Interventions}

\section{Section Editor: Daniel J. Feaster and} Allan E. Rodriguez

Behavioral Interventions for Adherence

Case Management for Linkage

Nurse-Delivered Interventions for Adherence

Retention in Care Interventions

\section{Behavioral Prevention}

\section{Section Editor: Lisa R. Metsch}

Behavioral Aspects of HIV Mother-to-Child Transmission
Behavioral Aspects of HIV Treatment as Prevention

Combination Approaches to HIV Prevention

Community Viral Load

Delayed Sexual Debut

Faith Based Interventions

Family Interventions

Female Condoms

HIV Prevention Efforts Within Substance Use

Disorder Treatment Settings

HIV Prevention and African Americans

HIV Prevention and Asians and Pacific Islanders

HIV Prevention and Hispanics

HIV Prevention and Labor Migration

HIV Prevention and Women

HIV Prevention for MSM

HIV Prevention for Serodiscordant Couples

HIV Prevention for Stimulant Using Men Who

Have Sex with Men

HIV Prevention in Persons 50 and Older

HIV Prevention in Transgender Persons

HIV Prevention in Youth

HIV Prevention in the Correctional System

HIV Testing and Counseling

HIV and Sexual Violence

Harm Reduction for Injection Drug Users

Housing as HIV Prevention

Male Condoms

Mass Media and HIV Prevention

Microfinance/Microenterprise Approaches to

HIV Prevention

Multilevel Interventions/Structural Approaches to

HIV Prevention 
Network Interventions

Peer-Based Intervention Approaches

Positive Health, Dignity, and Prevention (PHDP)

Preexposure Prophylaxis (PrEP)

Prevention Counseling and Other Strategies in the

HIV Care Setting

Prevention for People Living with HIV

Prevention of Alcohol-Related HIV Risk

Behavior

Sex Work and HIV Prevention

Translational Interventions (Intervention

Technology Transfer)

Use of Technology and Social Networking in HIV

Prevention

\section{Circumcision and AIDS}

Circumcision and AIDS

\section{Clinical: Opportunistic Infections}

\section{Section Editor: Hansjakob Furrer}

Bacterial Respiratory and Invasive Pneumococcal Infections and HIV

Chagas Disease and HIV

Cryptococcosis and HIV

Cryptosporidiosis

Cystoisosporiasis

Cytomegalovirus Infection and HIV

Hepatitis C Virus Infection and HIV

Leishmaniasis and HIV

M. avium Complex and Other Nontuberculous Mycobacteria and HIV

Microsporidiosis

Progressive Multifocal Leukoencephalopathy and HIV

Salmonellosis and Other Bacterial Enteric Infections and HIV

Talaromyces (Penicillium) marneffei and HIV

Toxoplasmosis in HIV-Infected Patients

Treatment of Histoplasmosis,

Coccidioidomycosis, and

Paracoccidioidomycosis in Patients with HIV

Infection

Tuberculosis and HIV

\section{Clinical: Opportunistic Malignancies}

\section{Section Editor: Robert Yarchoan}

AIDS-Related Primary Central Nervous System

Lymphoma

Anal Cancer

Burkitt and Burkitt-Like Lymphoma

Cervical Cancer and HIV

Conjunctival Carcinoma

Diffuse Large B-Cell Lymphoma

Epidemic Kaposi Sarcoma, Pathogenesis and Presentation

Epidemiology of AIDS-Defining Malignancies

Epidemiology of Non-AIDS-Defining

Malignancies

Epstein-Barr Virus (EBV)

HIV Cancers in Resource-Limited Regions

HIV-Associated Cancers

Hepatocellular Carcinoma in HIV-Positive Patients

Hodgkin Lymphoma in Patients with HIV Infection Human Papillomavirus (HPV)

Kaposi Sarcoma-Associated Herpesvirus

(KSHV) or Human Herpesvirus 8 (HHV-8)

Lung Cancer

Malignancies in Children with HIV Infection

Management of AIDS-Related Kaposi's Sarcoma

Merkel Cell Carcinoma and Other HIV-

Associated Skin Cancers

Merkel Cell Polyomavirus (MCV)

Multicentric Castleman Disease

Other HPV-Associated Cancers (Oropharyngeal and Penile)

Plasmablastic Lymphoma

Presentation and Pathogenesis of B-Cell Lymphoid Cancers Associated with HIV Infection

Primary Effusion Lymphoma

Stem Cell Transplantation

cART and Supportive Care for HIV-Associated Malignancies

\section{Epidemiology: Global Epidemiology}

\section{Section Editor: Sten H. Vermund}

Antiretroviral Medications, Adult Care, and Treatment 
Behavioral Science Highlights of Evidence and Research

Blood and Blood Product Donors and Recipients in China, Epidemiology of HIV/AIDS

Children, Care and Treatment

Children, Epidemiology of HIV/AIDS

Clinical Ethics in HIV/AIDS Prevention, Care, and Research

Eastern Europe and Central Asia, Specific Characteristics of HIV/AIDS Epidemic

Elderly: Epidemiology of HIV/AIDS

Female, Male and Transgender Sex Workers, Epidemiology of HIV/AIDS

Gay men and other Men who have sex with men (MSM), Epidemiology of HIV/AIDS Introduction

HIV Counseling and Testing, Prevention of HIV Health Care Workers, Epidemiology of HIV/ AIDS

Healthcare Workers, Shortage and Task Shifting of Jail and Prison Populations: Epidemiology of HIV/AIDS

Latin America and the Caribbean: Specific Characteristics of the HIV/AIDS Epidemic

Non-injecting Drug Users, Epidemiology of HIV/ AIDS

Pregnant Women: Care and Treatment

Preventing Mother-to-Child Transmission (PMTCT): Prevention of HIV

Prevention Clinical Trials: Highlights of Evidence and Research

South, Southeast, and East Asia-Specific Characteristics of HIV/AIDS Epidemic

Surveillance Case Definition

Women, Epidemiology of HIV/AIDS

\section{Epidemiology: HIV-2}

\section{Section Editor: Sarah L. Rowland-Jones}

Antibody Response to HIV-2

Antiretroviral Therapy and Drug Resistance in HIV-2 Infection

Cellular Immune Response to HIV-2 Infection Epidemiology of HIV-2 Infection in West Africa HIV-2 Diagnosis and Viral Load Measurements HIV-2 Envelope: Structure, Diversity, and Evolution
HIV-2 Infection in Europe, Epidemiology of HIV-2 Infection: The Role of Immune Activation in Pathogenesis

HIV-2 Neurological Manifestations

HIV-2 Transmission

HIV-2, Phylogeographic Insights into the Origins and Epidemic History

HIV-2: Lessons from the Dakar Cohort

Immunogenetics of HIV-2 Infection

Interactions Between HIV-2 and Host Restriction Factors

Molecular Biology of HIV-2

Natural History and Clinical Features of HIV-2 Infection

Natural Killer Cells Function and Innate Immunity in HIV-2 Infection

Recombinant Forms of HIV-2

Role of Dendritic Cells in HIV-2 Pathogenesis TRIM5 Alpha and HIV-2 Infection

Update on HIV-1 and HIV-2 Dual Infection

\section{Molecular Virology: Cellular Cofactors \& Cellular Restrictions}

Section Editor: Zeger Debyser

Acetylation

Actin

Arp2/3

BAF (BANF1)

CRM1

CXCR4, Coreceptors

Cellular Cofactors for HIV-1 Transcription

Cellular Cofactors of HIV as Drug Targets

Cofilin, Trafficking

Cyclophilin A and HIV-1 Replication

DDX3, Cofactors, and RNA Export

HIV-1 Assembly Cofactors

Identification and Validation of HIV Cofactors

Integrin Alpha4beta7

Nuclear Import: HIV-1 Goes NUPs

PIMT/TGS1

Role of Histone Deacetylases 1 and Yin Yang 1

Protein in Proviral Latency

Role of LEDGF/p75 in Cell Biology and Disease Pathogenesis

Role of Transportin-SR2 (Transportin-3, TRNSR2, TNPO3) in HIV Replication 


\section{Molecular Virology: Cellular Restriction Factors}

Section Editor: Monsef Benkirane and Nadine Laguette

APOBEC3F/G and Vif: Action and Counteractions Autophagy and HIV Infection

Counteraction of SAMHD1 by Vpx

HIV “Auxiliary" Proteins

Inflammasome and HIV

MX2 and HIV-1 Restriction

$\mathrm{Nef} / \mathrm{Env} / \mathrm{Vpu} / \mathrm{Tetherin}$

Overcoming the Transcriptional Block: The

HIV-1 Tat Auxiliary Protein

PKR and HIV Replication

Pyroptosis in HIV Infection: HIV Offense Meets a

Fiery Host Defense

SLX4 Complex and HIV Replication

TRIM Protein Family and Viral Restriction

TRIM5alpha

Viral Fitness in Hosts

Virus-Host Evolution and Positive Selection

\section{Molecular Virology: Life Cycle}

\section{Section Editor: Frank Kirchhoff}

Attachment/Binding

Budding

Fusion

HIV Life Cycle: Overview

HIV-1 Maturation

HIV-1 Rev Expression and Functions

HIV-1 Virion Structure

Integration

Macrophage-Specific Aspects of HIV-1 Infection

Reverse Transcriptase-Catalyzed HIV-1 DNA

Synthesis

Tat Expression and Function

Transcription (Initiation, Regulation, Elongation)

Uncoating and Nuclear Entry

Virus Assembly

Natural History \& Pathogenesis: Host Genetics

\section{Section Editor: Amalio Telenti}

Host Genetics and Genomics

KIR Locus Variation

MHC Locus Variation

Systems Biology

\section{Natural History \& Pathogenesis: Immune}

Pathogenesis

\section{Section Editor: Mathias Lichterfeld}

Cardiovascular Complications

Cell-Intrinsic Immunity

Cellular and Soluble Immune Activation Markers in HIV-Infected Subjects

Central Memory CD4 T Cells

Chronic Immune Activation in HIV

Collagen Deposition and Fibrosis in the

Lymphatic Tissues of HIV-1 Infected

Individuals

Gamma Delta T Cells

HIV Antigen Processing and Presentation

HIV Infection, Immune-Based Interventions for

HIV and SIV, B-Cell Responses to

HIV and SIV, CD4 T-Cell Responses to

HIV and SIV, CD8 T Cell Responses to

HIV-1 Mutational Escape from Host Immunity

HIV-Associated Immune Exhaustion

Immune Activation and HIV Transmission

Immunological Responses to Antiretroviral Therapy

Immunology of Latent HIV Infection

Immunopathogenesis of HIV

Coinfections

Inflammatory Cytokines

Long-Term Nonprogressors and Elite Controllers

Lymphocyte Apoptosis

Macrophages in HIV Immunopathogenesis

Microbial Translocation

Mucosal Immunity to HIV-1

NKT Cells: Bridging Innate and Adaptive Immunity

Neuro-AIDS, Immunopathogenesis of

Nonpathogenic SIV Infection of Sooty

Mangabeys

Overview: Immunopathogenesis

PD-1

Post-treatment Controllers

Role of Regulatory T Cells During HIV

Infection

SIV Infection in Mandrills

SIV Infection of African Green Monkeys

SIVmac Infection of Macaques, Immunopathogenesis of 
T Follicular Helper Cells in HIV Infection

T Memory Stem Cells

T-Cell Homeostasis

Th17 Cells

Thymic Function

Tim-3

Viremic Nonprogressors

Natural History \& Pathogenesis: Transmission

\section{Section Editor: William A. Paxton}

Dendritic Cell Interactions with HIV-1 Envelope Glycoprotein: Implications for Preventing Transmission

HIV Transmission in Female Commercial Sex Workers and Host Protective Factors

HIV-1 Preexposure Prophylaxis

HIV-1 Prevention Using Live-Attenuated Vaccines

HIV-1 Transmission Blocking Microbicides

HIV-1 Transmission: Influence of Bodily Secretions

Identifying Opportunities to Block HIV-1 Transmission in the Female Genital Tract

Inhibition of HIV-1 Spread: Cell-Free Versus Cell-Cell

MTCT HIV-1 Transmission Update:

Transmission Routes and Mechanisms

Mother-to-Child Transmission of HIV-1: Role of Receptor Usage and Target Cells

Natural Killer Cells and Their Role in Preventing HIV-1 Transmission

Non-neutralizing Antibody Responses and Protection Against HIV-1

Nonhuman Primate Models of HIV Transmission

Origin and Distribution of HIV-1 Subtypes

Preventing HIV-1 Transmission Through Vaccine-Induced Immune Responses

Preventing Mother-to-Child Transmission of HIV-1

Risk of Sexual HIV-1 Transmission: Coinfections Associated with Risk

Role of Antibodies in HIV Transmission

Transmission HIV-2: Origin, Epidemiology, and Phenotype

Tropism and Properties of the HIV-1 Envelope Glycoprotein in Transmission

\section{NeuroAIDS}

\section{Section Editor: Dennis L. Kolson}

Comorbidity: Opioids

Comorbidity: Progressive Multifocal Leukoencephalopathy

Differential Diagnosis of HIV-Associated Neurocognitive Disorders

Global NeuroAIDS

HAND Adjunctive Therapies: Reversing Neuronal Injury

HIV Neurocognitive Diagnosis, Natural History, and Treatment

Long-Acting Nanoformulated Antiretroviral Therapy

Medication Adherence and HIV-Associated Neurocognitive Disorders (HAND)

NeuroAIDS in Resource-Poor Settings, Assessment, and Treatment of

Neurocognitive Functioning in HIV-infected Substance Users

Neurocognitive Outcomes in HIV-Infected Children and Adolescents

Neuroinflammation and HAND: Therapeutic Targeting

Neuropsychological Testing in HIV-Infected Individuals

Neurotoxic Consequences of Antiretroviral Therapies

Overview of HIV CNS Infection

\section{Treatment}

\section{Section Editor: Marshall J. Glesby}

Antiretroviral Therapy for HIV-Infected Infants, Children, and Adolescents in Resource-Rich Settings

Antiretroviral Therapy: When to Start

Antiretroviral Treatment in Resource-Limited Settings

Entry Inhibitors

Initial Antiretroviral Regimens

Integrase Inhibitors

Maturation Inhibitor

NRTIs

Non-Nucleoside Reverse Transcriptase Inhibitors for Treatment of HIV Infection 
Prevention of Mother-to-Child Transmission of AIDS

Protease Inhibitor

Treatment Failure and Resistance

\section{Vaccines}

Vaccine Efforts Against AIDS 


\section{About the Editors-in-Chief}

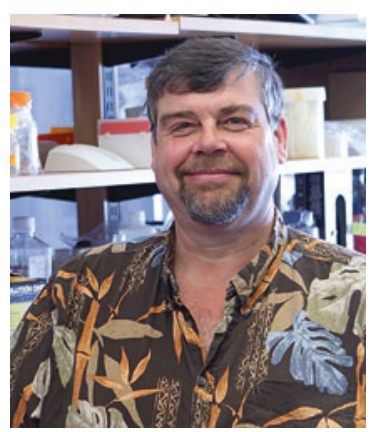

Thomas J. Hope Department of Cell and Molecular Biology, Northwestern University, Robert H. Lurie Medical Research Center, Chicago, IL, USA

Thomas J. Hope is currently a Professor at Northwestern University in the Departments of Cell and Molecular Biology and Obstetrics and Gynecology in the Feinberg School of Medicine and Professor in Biomedical Engineering in the Robert R. McCormick School of Engineering in Chicago, IL. He received his Ph.D. from the University of California, Berkeley, in Immunology. Tom began to study HIV in 1988, during his postdoctoral training at UC, San Francisco. He was one of the pioneers to utilizing the methods of cell biology to study HIV. In 2004, Tom was selected as an Elizabeth Glaser Scientist. For more than two decades, Tom has combined this cell biology approach, supplemented with molecular biology, biochemistry, and genetics, to study various aspects of HIV from virus entry, uncoating, and assembly to defining the earliest steps of sexual transmission and developing interventions to decrease transmission. Tom is currently the Editor-in-Chief of AIDS Research and Human Retroviruses, a Section Editor of PLoS Pathogens, serves on editorial boards and NIH/NIAID review panels, is a past co-organizer of HIVR4P, Keystone HIV, and Cold Spring Harbor retroviruses, and currently presents and teaches worldwide. 


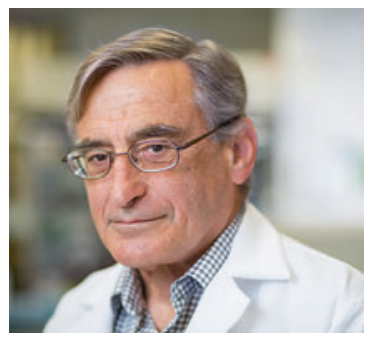

Dr. Douglas D. Richman VA San Diego Healthcare System and University of California San Diego, La Jolla, CA, USA

Dr. Douglas D. Richman received his A.B. from Dartmouth College and went on to receive his M.D. at Stanford University where he completed his residency. He was a Research Associate in the Laboratory of Infectious Diseases at the NIH's National Institute of Allergy and Infectious Diseases and Clinical Fellow in the Division of Infectious Diseases, Beth Israel Hospital and Children's Hospital Medical Center of Harvard.

Dr. Richman joined the University of California, San Diego (UCSD), in 1976 and is currently Distinguished Professor of Pathology and Medicine and holds the Florence Seeley Riford Chair in AIDS Research. He is Director of the Center for AIDS Research at UCSD, and he attends in infectious diseases at the San Diego VA Healthcare System. He is a Fellow of the American Association for the Advancement of Science, the American Association of Physicians, and the Infectious Disease Society of America.

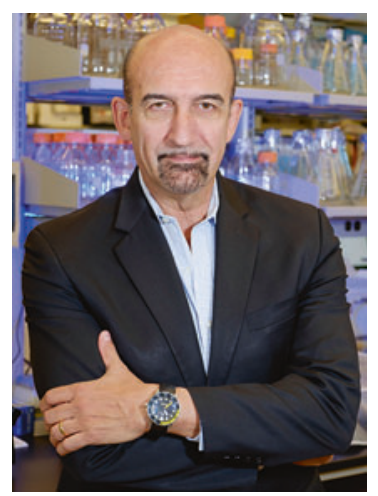

Dr. Mario Stevenson Department of Medicine, University of Miami Leonard M. Miller School of Medicine, Miami, FL, USA

Dr. Mario Stevenson is currently Professor in the Department of Medicine, Chief of the Division of Infectious Diseases, Director of the University of Miami AIDS Institute, Director of the Global Virus Network at the University of Miami, and Co-Director of the Center for AIDS Research. Prior to 2011, Dr. Stevenson was the David Freelander Chair for AIDS Research at the University of Massachusetts Medical School and the Director of the Center for AIDS Research at that institution. Dr. Stevenson obtained his B.Sc. (1980) 
and Ph.D. (1984) in Glasgow, Scotland. Dr. Stevenson is a molecular virologist who has been working on the viral etiology of AIDS for over 30 years. Dr. Stevenson has provided insight into the mechanisms regulating HIV replication, persistence, and disease pathogenesis. Dr. Stevenson has served as Chair of the HIV AIDS Virology Study Section at the National Institutes of Health, Chair of the Scientific Advisory Board of the National AIDS Conference (CROI), and has served on the NIH Office of AIDS Research that sets AIDS research directives. From 2006 to 2015, he served as Chair of the Scientific Advisory Board and a member of the Board of Trustees of the American Foundation for AIDS Research (amfAR) and a scientific board member of the Elizabeth Glaser Pediatric AIDS Foundation. Dr. Stevenson is a recipient of Harvard Medical School's Shipley Lectureship, recipient of the Gertrude Elion Award, Schally Research Award, and Barcelona's IRSI-Caixa Award for accomplishments in AIDS research. 


\section{Editorial Advisory Board}

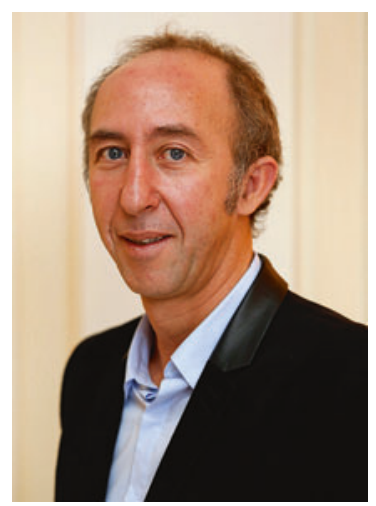

Monsef Benkirane Institut de Génétique Humaine, CNRS, Université de Montpellier, UMR9002, Laboratoire de Virologie Moléculaire, Montpellier, France

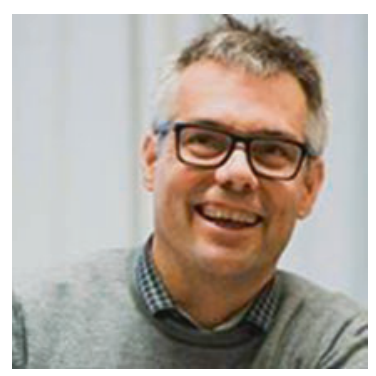

Zeger Debyser Laboratory for Molecular Virology \& Gene Therapy, Center for Molecular Medicine - Faculty of Medicine - KU Leuven, Leuven, Flanders, Belgium 


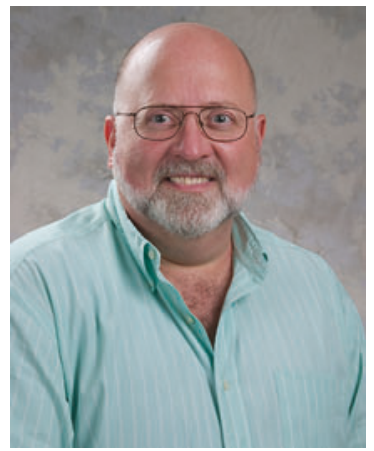

Daniel J. Feaster Division of Biostatistics, Department of Public Health, University of Miami Miller School of Medicine, Miami, FL, USA

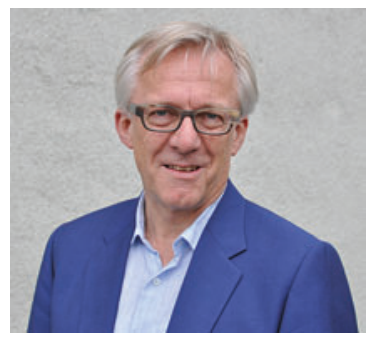

Hansjakob Furrer Department of Infectious Diseases, Bern University Hospital, University of Bern, Bern, Switzerland

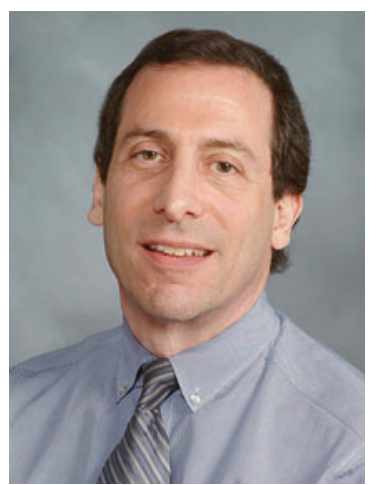

Marshall J. Glesby Division of Infectious Diseases, Department of Medicine, Weill Cornell Medical College, New York, NY, USA 


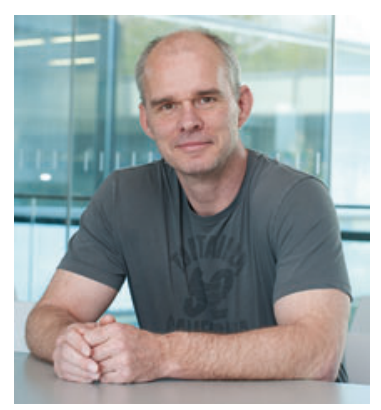

Frank Kirchhoff Institute of Molecular Virology, Ulm University Medical Center, Ulm, Germany

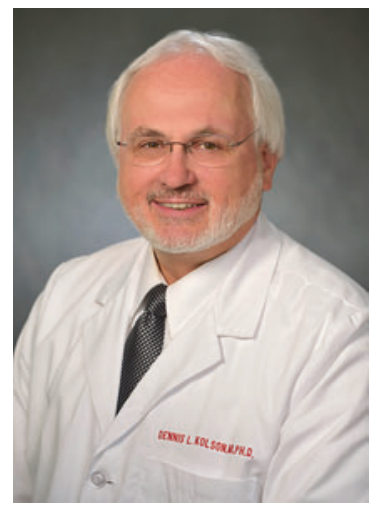

Dennis L. Kolson Department of Neurology, University of Pennsylvania, Philadelphia, PA, USA

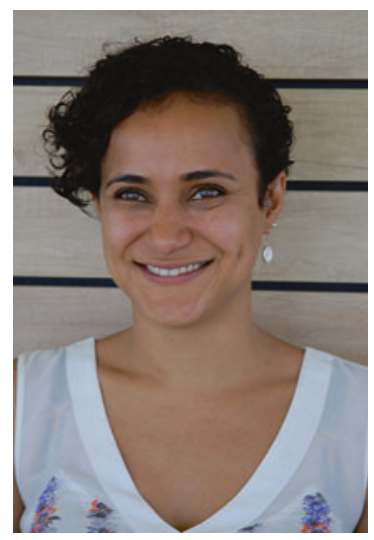

Nadine Laguette Institute of Human Genetics, Molecular Basis of CancerRelated Inflammation Laboratory, CNRS UPR1142, Montpellier, France 


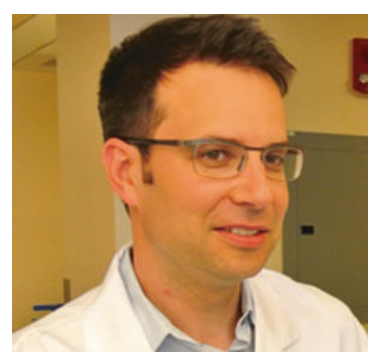

Mathias Lichterfeld Infectious Disease Division, Brigham and Women's Hospital, Boston, MA, USA

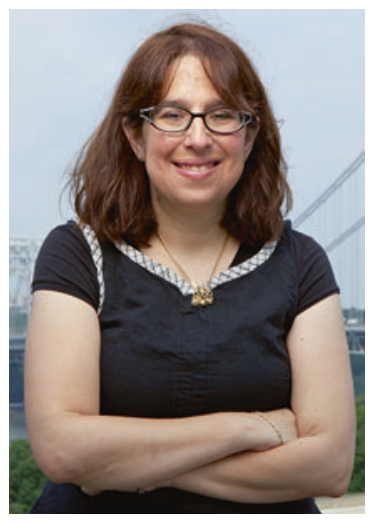

Lisa R. Metsch Department of Sociomedical Sciences, Mailman School of Public Health, Columbia University, New York, NY, USA

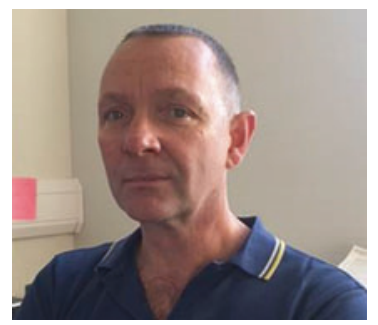

William A. Paxton Department of Clinical Infection, Microbiology and Immunology, Institute of Infection and Global Health, University of Liverpool, Liverpool, UK 


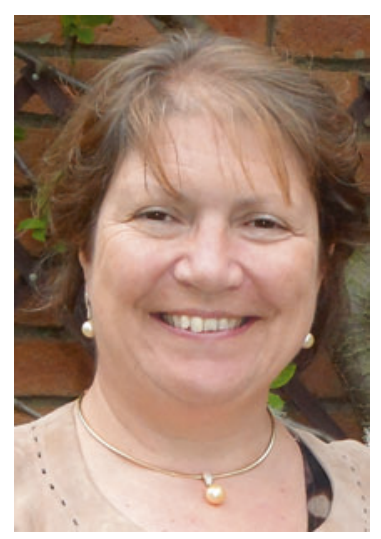

Sarah L. Rowland-Jones Nuffield Department of Medicine, NDM Research Building, University of Oxford, Headington, Oxford, UK

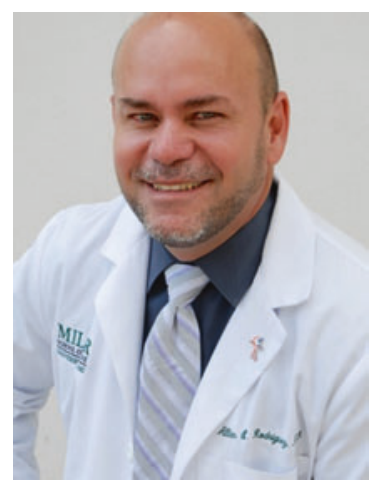

Allan E. Rodriguez University of Miami, Miller School of Medicine, Miami, FL, USA

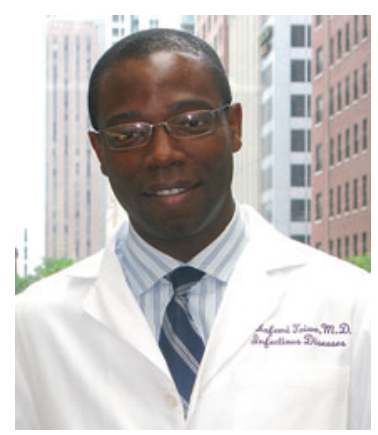

Babafemi O. Taiwo Division of Infectious Diseases, Northwestern University Feinberg School of Medicine, Chicago, IL, USA 


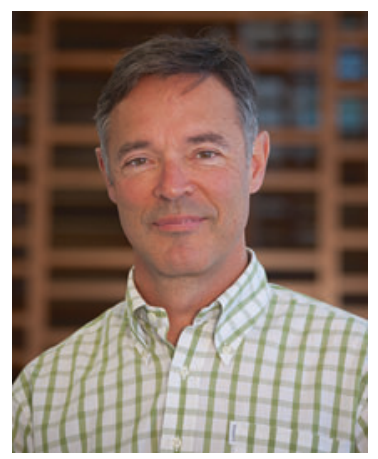

Amalio Telenti The J Craig Venter Institute, La Jolla, CA, USA

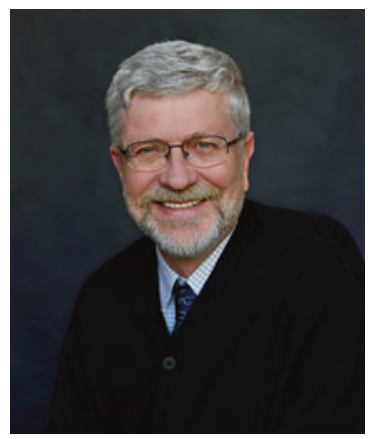

Sten H. Vermund Yale School of Public Health, New Haven, CT, USA

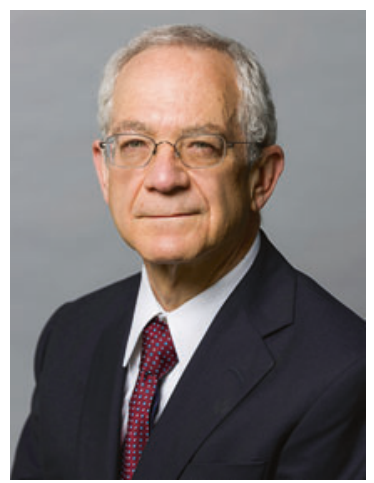

Robert Yarchoan Center for Cancer Research, National Cancer Institute, Bethesda, MD, USA 


\section{Contributors}

Salim S. Abdool Karim CAPRISA - Centre for the AIDS Programme of Research in South Africa, University of KwaZulu-Natal, Durban, South Africa Department of Epidemiology, Mailman School of Public Health, Columbia University, New York, NY, USA

Quarraisha Abdool Karim CAPRISA - Centre for the AIDS Programme of Research in South Africa, University of KwaZulu-Natal, Durban, South Africa Department of Epidemiology, Mailman School of Public Health, Columbia University, New York, NY, USA

Ana Abecasis Global Health and Tropical Medicine, Institute for Hygiene and Tropical Medicine, Universidade Nova de Lisboa, Lisbon, Portugal International Public Health and Biostatistics Unit, Institute for Hygiene and Tropical Medicine, Universidade Nova de Lisboa, Lisbon, Portugal

David M. Aboulafia Division of Hematology and Oncology, Virginia Mason Medical Center, Seattle, WA, USA

Division of Hematology, University of Washington, Seattle, WA, USA

Angela Aidala Sociomedical Sciences, Mailman School of Public Health, Columbia University, New York, NY, USA

Christopher Aiken Department of Pathology, Microbiology and Immunology, Vanderbilt University School of Medicine, Nashville, TN, USA

Sol Aldrete Division of Infectious Diseases, Emory University School of Medicine, Atlanta, GA, USA

Grace M. Aldrovandi Division of Infectious Disease, The Saban Research Institute, Children's Hospital Los Angeles, Los Angeles, CA, USA

Jamal Alfaisal Centre d'études d'agents Pathogènes et Biotechnologies pour la Santé (CPBS), CNRS-UMR, Montpellier, France

Mohammed K. Ali Hubert Department of Global Health, Rollins School of Public Health, Emory University, Atlanta, GA, USA

Emory University School of Medicine, Atlanta, GA, USA 
Awatef Allouch INSERM U1030 - LabEx LERMIT, Institut Gustave Roussy, Villejuif, France

Míriam J. Alvarez-Martínez Microbiology Service, Hospital ClínicISGLOBAL, University of Barcelona, Barcelona, Spain

Richard Ambinder Department of Oncology, Division of Hematologic Malignancies, Johns Hopkins University School of Medicine, Baltimore, MA, USA

Juan Ambrosioni Infectious Diseases Service, Hospital Clínic-IDIBAPS, University of Barcelona, Barcelona, Spain

Neil M. Ampel Department of Medicine, Medical Service (1-111), University of Arizona College of Medicine and the Southern Arizona Veterans Affairs Health Care System (SAVAHCS), Tucson, AZ, USA

Ronald Anderson Emeritus Professor, Institute of Cellular and Molecular Medicine, Department of Immunology, Faculty of Health Sciences, University of Pretoria, Pretoria, South Africa

Sophie M. Andrews Nuffield Department of Medicine, University of Oxford, Oxford, Oxfordshire, UK

Nadav Antebi Sociomedical Sciences, Mailman School of Public Health, Columbia University, New York, NY, USA

Cristian Apetrei Center for Vaccine Research and Department of Microbiology and Molecular genetics, School of Medicine, University of Pittsburgh, Pittsburgh, PA, USA

Victor Appay Centre d'Immunologie et des Maladies Infectieuses (CIMIParis), Sorbonne Universités, UPMC Univ Paris 06, CR7, Paris, France

INSERM, U1135, CIMI-Paris, Paris, France

Accursio Augello Division of Medical Oncology A, National Cancer Institute, Aviano, PN, Italy

Brigitte Autran Laboratory Immunity and Infection, UPMC University Paris VI, Paris, France

Laboratory Immunity and Infection, INSERM U1135, Paris, France

Andrew D. Badley Division of Infectious Diseases, Mayo Clinic, Rochester, MN, USA

Division of Molecular Medicine, Mayo Clinic, Rochester, MN, USA

Ester Ballana IrsiCaixa, Hospital Universitari Germans Trias i Pujol, Universitat Autònoma de Barcelona, Badalona, Spain

Stefan D. Baral Johns Hopkins Bloomberg School of Public Health, Center for Public Health and Human Rights, Department of Epidemiology, Baltimore, MD, USA

Linda Barlow-Mosha Makerere University Johns Hopkins University Research Collaboration, Kampala, Uganda 
John A. Bartlett Duke Global Health Institute, Durham, NC, USA

Kilimanjaro Christian Medical Center, Moshi, Tanzania

Duke University Medical Center, Durham, NC, USA

Arman Bashirova Ragon Institute of MGH, MIT and Harvard, Boston, MA, USA

Julien Batisse Architecture et Réactivité de l'ARN, UPR 9002 CNRS, Institut de Biologie Moléculaire et Cellulaire du CNRS, Université de Strasbourg, Strasbourg, France

IGBMC, CNRS UMR 7104, Illkirch, France

Cheryl Baxter CAPRISA - Centre for the AIDS Programme of Research in South Africa, University of KwaZulu-Natal, Durban, South Africa

Karen Beckerman Department of Obstetrics and Gynecology and Women's Health, Division of Maternal-Fetal Medicine, Albert Einstein College of Medicine, Bronx, NY, USA

Consuelo M. Beck-Sagué Department of Health Promotion and Disease Prevention, Robert Stempel College of Public Health and Social Work, Florida International University, Miami, FL, USA

Serge Benichou Inserm U1016, Institut Cochin, Paris, France

CNRS, UMR8104, Paris, France

Sorbonne Paris-Cité, Université Paris-Descartes, Paris, France

Constance A. Benson Division of Infectious Diseases, Department of Medicine, The University of California, San Diego, CA, USA

Joan Berenguer Radiology Service CDIC, Hospital Clinic-IDIBAPS, University of Barcelona, Barcelona, Spain

Massimiliano Berretta Department of Medical Oncology A, National Cancer Institute, Aviano, PN, Italy

Neil J. Berry Division of Virology, National Institute for Biological Standards and Control, South Mimms, Hertfordshire, UK

Chris Beyrer Johns Hopkins Bloomberg School of Public Health, Center for Public Health and Human Rights, Department of Epidemiology, Baltimore, MD, USA

Mallika Bhandarkar In care of Dr. Diana Hernandez, Columbia University, New York, NY, USA

Kishor Bhatia AIDS Malignancy Program, Office of HIV and AIDS Malignancy and Infections and Immunoepidemiology Branch, National Cancer Institute, Bethesda, MD, USA

Nisha S. Bhatia Department of Neurology, University of California San Francisco, San Francisco, CA, USA 
Martine Biard-Piechaczyk Centre d'études d'agents Pathogènes et Biotechnologies pour la Santé (CPBS), CNRS-UMR, Montpellier, France

Fabien P. Blanchet Centre d'études d'agents Pathogènes et Biotechnologies pour la Santé (CPBS), CNRS-UMR, Montpellier, France

Julià Blanco Fundacio irsiCaixa, Institut de Recerca Germans Trias I Pujol (IGTP), Barcelona, Spain

Universitat de Vic (UVIC-UCC), Vic, Spain

Joel N. Blankson Division of Infectious Diseases, Department of Medicine, Johns Hopkins University School of Medicine, Baltimore, MD, USA

William A. Blattner Division of Epidemiology and Prevention, Institute of Human Virology, University of Maryland School of Medicine, Baltimore, USA

Jill Blumenthal Division of Infectious Diseases, Department of Medicine, University of California San Diego, San Diego, CA, USA

Geneviève Boily-Larouche Medical Microbiology Department, University of Manitoba, Winnipeg, MB, Canada

Nell G. Bond Department of Microbiology and Immunology, Tulane University School of Medicine; Tulane National Primate Research Center, Covington, LA, USA

José A. M. Borghans Laboratory of Translational Immunology, Department of Immunology, University Medical Center Utrecht, Utrecht, The Netherlands

Steven E. Bosinger Division of Microbiology and Immunology, Yerkes National Primate Research Center, Emory University, Atlanta, GA, USA

Fadila Bouamr Laboratory of Molecular Microbiology, National Institute of Allergy and Infectious Diseases, National Institutes of Health, Bethesda, MD, USA

Julie Boucau Ragon Institute of MGH, MIT and Harvard, Harvard Medical School, Cambridge, MA, USA

S. Bouchat Institute for Molecular Biology and Medicine (IBMM), Université Libre de Bruxelles (ULB), Service of Molecular Virology, Gosselies, Belgium

David R. Boulware Division of Infectious Diseases and International Medicine, Department of Medicine, University of Minnesota, Minneapolis, MN, USA

Christelle Brégnard Institute of Human Genetics, Molecular Basis of Cancer-Related Inflammation Laboratory, CNRS UPR1142, Montpellier, France

Jason M. Brenchley Laboratory of Molecular Microbiology, NIAID, National Institutes of Health, Bethesda, MD, USA 
Bruce J. Brew Neurology and HIV Departments Sydney, St. Vincent's Hospital, Sydney, NSW, Australia

Peter Duncan Neurosciences Unit, St. Vincent's Centre for Applied Medical Research, Sydney, NSW, Australia

Lauren Brinkley-Rubinstein Department of Social Medicine/Center for Health Equity Research, University of North Carolina at Chapel Hill, Chapel Hill, NC, USA

University of North Carolina at Chapel Hill, Chapel Hill, NC, USA

Kristina Broliden Department of Medicine Solna, Karolinska Institute, Center for Molecular Medicine, Karolinska University Hospital, Stockholm, Sweden

Felicia A. Browne Substance Use, Gender and Applied Research, RTI International, Research Triangle Park, NC, USA

Zabrina L. Brumme Faculty of Health Sciences, Simon Fraser University, Burnaby, BC, Canada

British Columbia Centre for Excellence in HIV/AIDS, Vancouver, BC, Canada

Francoise Brun-Vezinet Laboratoire de Virologie, Service de Microbiologie, Bichat-Claude Bernard Hospital and University, Paris, France

Robert W. Buckheit III Virus-Cell Interaction Section, National Cancer Institute at Frederick, HIV Drug Resistance Program, Frederick, MD, USA

Tricia H. Burdo Department of Biology, Boston College, Chestnut Hill, MA, USA

Adam Burgener Department of Medical Microbiology, University of Manitoba, Winnipeg, MB, Canada

Leah A. Burke Division of Infectious Diseases, Weill Cornell Medicine, New York, NY, USA

Frederic D. Bushman Department of Microbiology, University of Pennsylvania, Perelman School of Medicine, Philadelphia, PA, USA

Jennifer Butler Eastern Europe Central Asia Regional Office, United Nations Population Fund, Istanbul, Turkey

Maria J. Buzon Ragon Institute of MGH, MIT and Harvard, Boston, MA, USA

Infectious Disease Division, Massachusetts General Hospital, Boston, MA, USA

Bruno Cacopardo Department of Clinical and Molecular Biomedicine, Division of Infectious Diseases, University of Catania, Catania, Italy 
Andrea Calcagno Unit of Infectious Diseases, Department of Medical Sciences, University of Torino, Torino, Italy

Donald A. Calsyn Alcohol and Drug Abuse Institute, University of Washington, Seattle, WA, USA

Department of Psychiatry and Behavioral Sciences, University of Washington, Seattle, WA, USA

Guislaine Carcelain Laboratory Immunity and Infection, UPMC University Paris VI, Paris, France

Laboratory Immunity and Infection, INSERM U1135, Paris, France

James G. Carlucci Vanderbilt Institute for Global Health and Division of Pediatric Infectious Diseases, Vanderbilt University, Nashville, TN, USA

Mary Carrington Cancer and Inflammation Program, Laboratory of Experimental Immunology, Leidos Biomedical Research Inc., Frederick National Laboratory for Cancer Research, Frederick, MD, USA

Ragon Institute of MGH, MIT and Harvard, Boston, MA, USA

Kaitlin B. Casaletto SDSU/UCSD Joint Doctoral Program in Clinical Psychology, San Diego State University/University of California, San Diego, CA, USA

Corey Casper Vaccine and Infectious Disease, Public Health Sciences and Clinical Research Divisions, Departments of Medicine, Epidemiology and Global Health, Fred Hutchinson Cancer Research Center and the University of Washington, Seattle, WA, USA

Amanda D. Castel Department of Epidemiology and Biostatistics, The George Washington University Milken Institute School of Public Health, Washington, DC, USA

Alice Cates Department of Epidemiology and Biostatistics, The George Washington University Milken Institute School of Public Health, Washington, DC, USA

Mariangela Cavarelli Viral Evolution and Transmission Unit, Division of Immunology, Transplantation and Infectious Diseases, San Raffaele Scientific Institute, Milan, Italy

Anna Cereseto Centre for Integrative Biology, Laboratory of Molecular Virology, University of Trento, Trento, Italy

Giuliana Cerro Universidad Peruana Cayetano Heredia, Lima, Peru

Romanee Chaiwarith Division of Infectious Diseases, Faculty of Medicine, Chiang Mai University, Chiang Mai, Thailand

Andrew Chandranesan Infectious Diseases, Tufts University School of Medicine, Boston, MA, USA

Donald A. Calsyn: deceased. 
Christina C. Chang Department of Infectious Diseases, Alfred Hospital and Monash University, Melbourne, Australia

Centre for Biomedical Research, Burnet Institute, Melbourne, Australia

Zheng W. Chen Department of Microbiology and Immunology, Center for Primate Biomedical Research, University of Illinois College of Medicine Chicago, Chicago, IL, USA

Rémi Cheynier Université Paris Descartes, Paris, France

INSERM U1016, Paris, France

CNRS UMR8104, Paris, France

Benjamin H. Chi Department of Obstetrics and Gynecology, University of North Carolina at Chapel Hill, Chapel Hill, NC, USA

Carla J. Chibwesha Department of Obstetrics and Gynecology, University of North Carolina at Chapel Hill, Chapel Hill, NC, USA

Nyaradzo T. L. Chigorimbo-Murefu Institute of Infectious Disease and Molecular Medicine, Division of Immunology, Department of Pathology, University of Cape Town, Cape Town, South Africa

Youngjee Choi Department of Medicine, Washington University in St. Louis, St Louis, MO, USA

Nicolas Chomont Vaccine and Gene Therapy Institute of Florida, Port St Lucie, FL, USA

Claire A. Chougnet Department of Immunobiology, Cincinnati Children Hospital Medical Center, Cincinnati, OH, USA

Samson A. Chow Department of Molecular and Medical Pharmacology, UCLA AIDS Institute, UCLA School of Medicine, Los Angeles, CA, USA

Frauke Christ Laboratory for Molecular Virology and Gene Therapy, KU Leuven, Leuven, Flanders, Belgium

Rosanna Ciancia Division of Medical Oncology A, National Cancer Institute, Aviano, PN, Italy

Paola Cinque Department of Infectious Diseases, San Raffaele Scientific Institute, Milan, Italy

Angela Ciuffi Institute of Microbiology, University Hospital Center and University of Lausanne, Lausanne, Switzerland

Paul Clapham Program in Molecular Medicine, University of Massachusetts Medical School, Worcester, MA, USA

Department of Microbiology and Physiological Systems, University of Massachusetts Medical School, Worcester, MA, USA

C. Brendan Clark Department of Psychiatry, Substance Abuse Center, University of Alabama at Birmingham, Birmingham, AL, USA 
Kiera Clayton Department of Immunology, University of Toronto, Toronto, ON, Canada

David B. Clifford Departments of Medicine and Neurology, Washington University in St. Louis, St Louis, MO, USA

Elizabeth F. Closson The Fenway Institute, Fenway Health, Boston, MA, USA

The London School of Hygiene and Tropical Medicine, London, UK

Ronald G. Collman Perelman School of Medicine, Department of Medicine, Division of Pulmonary, Allergy and Critical Care, University of Pennsylvania, Philadelphia, PA, USA

Ryan J. Conrad Gladstone Institute of Virology and Immunology, University of California, San Francisco, San Francisco, CA, USA

Christa L. Cook University of Florida, College of Nursing, Gainesville, FL, USA

Cecilia T. Costiniuk Department of Medicine, Divisions of Infectious Diseases/Chronic Viral Illness Service and Lachine Hospital, McGill University Health Centre, Montreal, QC, Canada

Robert Craigie Laboratory of Molecular Biology, National Institute of Diabetes and Digestive and Kidney Diseases, National Institutes of Health, Bethesda, MD, USA

Megan Crane Department of Infectious Diseases, Alfred Hospital and Monash University, Melbourne, Australia

Centre for Biomedical Research, Burnet Institute, Melbourne, Australia

Natalie D. Crawford Behavioral Sciences and Health Education, Rollins School of Public Health, Emory University, Atlanta, GA, USA

Nicole Crepaz Division of HIV/AIDS Prevention, The U.S. Centers for Disease Control and Prevention, Atlanta, GA, USA

Karen Cropsey Department of Psychiatry and Behavioral Neurobiology, University of Alabama at Birmingham, Birmingham, AL, USA

Stephanie A. Cross Department of Psychiatry, Perelman School of Medicine, University of Pennsylvania, Philadelphia, PA, USA

Nathan W. Cummins Division of Infectious Diseases, Mayo Clinic, Rochester, MN, USA

Eric S. Daar Division of HIV Medicine, Department of Medicine, HarborUCLA Medical Center, David Geffen School of Medicine at UCLA, Torrance, CA, USA

Kristina R. Dahlstrom Department of Head and Neck Surgery, The University of Texas MD Anderson Cancer Center, Houston, TX, USA 
Safiya George Dalmida Capstone College of Nursing, The University of Alabama, Tuscaloosa, AL, USA

Blossom Damania Program in Global Oncology, Lineberger Comprehensive Cancer Center, Department of Microbiology and Immunology, Center for AIDS Research, University of North Carolina, Chapel Hill, NC, USA

Moupali Das HIV/AIDS and Infectious Diseases, University of California San Francisco, San Francisco, CA, USA

Coralie F. Daussy Centre d'études d'agents Pathogènes et Biotechnologies pour la Santé (CPBS), CNRS-UMR, Montpellier, France

Melissa A. Davey-Rothwell Department of Health, Behavior and Society, Johns Hopkins Bloomberg School of Public Health, Baltimore, MD, USA

Jan Davidson-Moncada Medical Oncology Branch, Center for Cancer Research, National Cancer Institute (NCI), Bethesda, MD, USA

Paolo De Paoli Scientific Directorate, National Cancer Institute, Aviano, PN, Italy

Suresh de Silva Department of Veterinary Biosciences, Center for Retrovirus Research, The Ohio State University, Columbus, OH, USA

Zeger Debyser Laboratory for Molecular Virology \& Gene Therapy, Center for Molecular Medicine - Faculty of Medicine - KU Leuven, Leuven, Flanders, Belgium

Kathleen N. Deering Gender and Sexual Health Initiative, BC Centre for Excellence in HIV/AIDS, St. Paul's Hospital, Vancouver, BC, Canada

Division of AIDS, Department of Medicine, Faculty of Medicine, University of British Columbia, Vancouver, BC, Canada

Erin Dehon G. V. (Sonny) Montgomery VAMC, Jackson, MS, USA

Jack DeHovitz Department of Medicine, Division of Infectious Disease, SUNY Downstate Medical Center, Brooklyn, NY, USA

Ana María del Río González Department of Psychology, The George Washington University, Washington, DC, USA

Julie A. Denison Department of International Health, Johns Hopkins Bloomberg School of Public Health, Baltimore, MD, USA

Nina Derby Center for Biomedical Research, Population Council, New York, NY, USA

Sherry Deren Center for Drug Use and HIV Research, New York University College of Nursing, New York, NY, USA

Diane Descamps Laboratoire de Virologie, Service de Microbiologie, Bichat-Claude Bernard Hospital and University, Paris, France

Belete A. Desimmie Laboratory for Molecular Virology and Gene Therapy, KU Leuven, Leuven, Flanders, Belgium 
Ronald C. Desrosiers Department of Pathology, University of Miami Miller School of Medicine, Miami, FL, USA

Abby DiCarlo Department of Sociomedical Sciences, Mailman School of Public Health, Columbia University, New York, NY, USA

Stephanie M. Dillon Department of Medicine, Division of Infectious Diseases, University of Colorado Anschutz Medical Campus, Aurora, CO, USA

Dirk P. Dittmer Program in Global Oncology, Lineberger Comprehensive Cancer Center, Department of Microbiology and Immunology, Center for AIDS Research, University of North Carolina, Chapel Hill, NC, USA

Gilad Doitsh, Gladstone Institutes, Institute of Virology and Immunology, San Francisco, CA, USA

Antoine Douaihy Department of Psychiatry, University of Pittsburgh School of Medicine, Pittsburgh, PA, USA

Julia Drylewicz Laboratory of Translational Immunology, Department of Immunology, University Medical Center Utrecht, Utrecht, The Netherlands

Elena Dukhovlinova The University of North Carolina, Lineberger Cancer Center, Chapel Hill, NC, USA

Kieron Dunleavy Metabolism Branch, CCR, National Cancer Institute (NCI), Bethesda, MD, USA

Christine Durand Department of Medicine, Division of Infectious Diseases, Johns Hopkins University School of Medicine, Baltimore, MA, USA

Vincent Dussupt Laboratory of Molecular Microbiology, National Institute of Allergy and Infectious Diseases, National Institutes of Health, Bethesda, MD, USA

Jacques Dutrieux INSERM UMR-S 1124, Paris, France

Université Paris Descartes, Paris, France

Laura J. Edwards Division of Health and Environment, Abt Associates, Atlanta, GA, USA

Serge Paul Eholie Programme PACCI, Site ANRS, Abidjan, Côte d'Ivoire Service des Maladies Infectieuses et Tropicales, CHU de Treichville, Abidjan, Côte d'Ivoire

Département de Dermatologie-Infectiologie, Unité de Formation et de Recherche des Sciences Médicales, Abidjan, Côte d'Ivoire

Didier Koumavi Ekouevi Département de Santé Publique, Faculté des Sciences de la Santé, Université de Lomé (Togo), Lomé, Togo

Programme PACCI, Site ANRS, Abidjan, Côte d'Ivoire

Nabila El-Bassel Social Intervention Group, Columbia University School of Social Work, NY, USA 
Hila Elinav Department of Clinical Microbiology and Infectious Diseases, Hadassah Hebrew University Medical Center, Jerusalem, Israel

Sarah T. Elliot Perelman School of Medicine, Department of Medicine, Division of Pulmonary, Allergy and Critical Care, University of Pennsylvania, Philadelphia, PA, USA

Ronald J. Ellis Department of Neurosciences, University of California, San Diego, UCSD HNRC, San Diego, CA, USA

Joakim Esbjörnsson Department of Laboratory Medicine Malmö, Lund University, Malmö, Sweden

Department of Microbiology, Tumor and Cell Biology (MTC), Karolinska Institute, Stockholm, Sweden

Weather all Institute of Molecular Medicine, University of Oxford, Oxford, Great Britain

Lucile Espert Centre d'études d'agents Pathogènes et Biotechnologies pour la Santé (CPBS), CNRS-UMR, Montpellier, France

José A. Esté IrsiCaixa, Hospital Universitari Germans Trias i Pujol, Universitat Autònoma de Barcelona, Badalona, Spain

Yannine Estrada Department of Public Health Sciences, University of Miami, Miami, FL, USA

Lucie Etienne CIRI, Centre International de Recherche en Infectiologie, INSERM U1111 - CNRS UMR5308 - Université Lyon 1 - ENS de Lyon, Lyon, France

Jean-Louis Excler U.S. Military HIV Research Program, Bethesda, MD, USA

Theresa Exner HIV Center for Clinical and Behavioral Studies, New York State Psychiatric Institute, Columbia University Medical Center, New York, NY, USA

Claire E. Farel Institute for Global Health and Infectious Diseases, University of North Carolina, Chapel Hill, NC, USA

Nuno Rodrigues Faria Department of Microbiology and Immunology, Rega Institute for Medical Research, Leuven, KU, Belgium

Charles Feldman Charlotte Maxeke Johannesburg Academic Hospital and Faculty of Health Sciences, University of the Witwatersrand, Johannesburg, South Africa

Jacques Fellay School of Life Sciences, Ecole Polytechnique Fédérale de Lausanne, Lausanne, Vaud, Switzerland

Christine Fennema-Notestine Departments of Psychiatry and Radiology, University of California, San Diego, La Jolla, CA, USA 
Eva Maria Fenyö Department of Microbiology, Tumor and Cell Biology (MTC), Karolinska Institute, Stockholm, Sweden

Nicole Fischer Institute for Medical Microbiology, Virology and Hygiene, University Medical Center Hamburg-Eppendorf, Hamburg, Germany

Christina Fiske Division of Infectious Diseases, Vanderbilt University Medical Center, Nashville, TN, USA

Timothy Fitzgerald Center for Neural Development and Disease, Department of Neurology, University of Rochester Medical Center, Rochester, NY, USA

Annesa Flentje Department of Psychiatry, Community Health Systems, University of California, San Francisco, CA, USA

Department of Psychiatry, University of California, San Francisco, CA, USA

Courtney V. Fletcher Antiviral Pharmacology Laboratory and Department of Pharmacy Practice, College of Pharmacy, University of Nebraska Medical Center, Omaha, NE, USA

Division of Infectious Diseases, Department of Internal Medicine, University of Nebraska Medical Center, Omaha, NE, USA

Virginia A. Fonner Department of Psychiatry and Behavioral Sciences, Division of Global and Community Health, Medical University of South Carolina, Charleston, SC, USA

Keith Raymond Fowke Medical Microbiology Department, University of Manitoba, Winnipeg, MB, Canada

Department of Community Health Sciences, University of Manitoba, Winnipeg, Canada

Department of Medical Microbiology, University of Nairobi, Nairobi, Kenya

Mary Glenn Fowler Department of Pathology, Johns Hopkins University School of Medicine, Baltimore, MD, USA

Russell B. Foxall Unidade de Imunologia Clínica, Instituto de Medicina Molecular, Faculdade de Medicina, Universidade de Lisboa, Lisbon, Portugal

Nicholas Francella Perelman School of Medicine, Department of Medicine, Division of Pulmonary, Allergy and Critical Care, University of Pennsylvania, Philadelphia, PA, USA

Eric O. Freed HIV Drug Resistance Program, Frederick National Laboratory, Frederick, MD, USA

Martyn A. French School of Pathology and Laboratory Medicine, University of Western Australia, Perth, WA, Australia

Department of Clinical Immunology, Royal Perth Hospital and PathWest Laboratory Medicine, Perth, Australia

Rémi Fromentin Vaccine and Gene Therapy Institute of Florida, Port St Lucie, FL, USA 
Julie Christine Gaardbo Department of Infectious Diseases, University of Copenhagen, Rigshospitalet, Copenhagen, Denmark

Joel Gallant Southwest CARE Center, Santa Fe, NM, USA

Barbie K. Ganser-Pornillos Department of Molecular Physiology and Biological Physics, University of Virginia, Charlottesville, VA, USA

Xiaojiang Gao Cancer and Inflammation Program, Laboratory of Experimental Immunology, SAIC-Frederick, Inc., Frederick National Laboratory for Cancer Research, Frederick, MD, USA

Ragon Institute of MGH, MIT and Harvard, Boston, MA, USA

Justin Y. Garber Neurology and HIV Departments Sydney, St. Vincent's Hospital, Sydney, NSW, Australia

Anne Gatignol Virus-Cell Interactions Laboratory, Lady Davis Institute for Medical Research, Montréal, QC, Canada

Departments of Medicine and Microbiology and Immunology, McGill University, Montréal, QC, Canada

Harris A. Gelbard Neurology in the Center for Neural Development and Disease, School of Medicine and Dentistry, University of Rochester Medical Center, Rochester, NY, USA

Howard E. Gendelman Department of Pharmaceutical Sciences, University of Nebraska Medical Center, Omaha, NE, USA

Department of Pharmacology and Experimental Neuroscience, University of Nebraska Medical Center, Omaha, NE, USA

Meritxell Genescà Department of Mucosal Immunology, Institute for AIDS Research IrsiCaixa, Health Sciences Research Institute Germans Trias i Pujol Foundation, Badalona, Barcelona, Spain

Simonetta Gerevini Department of Nuroradiology, San Raffaele Scientific Institute, Milan, Italy

Jan Gerstoft Department of Infectious Diseases, University of Copenhagen, Rigshospitalet, Copenhagen, Denmark

Lavina Gharu Laboratory of Molecular Virology, International Centre for Genetic Engineering and Biotechnology (ICGEB), AREA Science Park, Trieste, Italy

Mauro Giacca Molecular Medicine Laboratory, International Centre for Genetic Engineering and Biotechnology (ICGEB), Trieste, Italy

Louisa Gilbert Social Intervention Group, Columbia University School of Social Work, NY, USA

Jody Glance Department of Psychiatry, University of Pittsburgh School of Medicine, Pittsburgh, PA, USA

Marshall J. Glesby Division of Infectious Diseases, Department of Medicine, Weill Cornell Medical College, New York, NY, USA 
Felisa A. Gonzales Department of Psychology, The George Washington University, Washington, DC, USA

Raul Gonzalez Department of Psychology, Florida International University, Miami, FL, USA

Geoffrey S. Gottlieb Division of Allergy and Infectious Diseases, Department of Medicine and Department of Global Health, University of Washington School of Medicine, Seattle, WA, USA

Eduardo Gotuzzo Instituto de medicina Tropical alexander von Humboldt, Universidad Peruana Cayetano Heredia, Lima, Peru

Caroline Goujon Centre d'études d'agents pathogènes et biotechnologies pour la santé, CNRS FRE3689 - UM, Montpellier, France

Erin Graves Vanderbilt University School of Medicine, Tennessee Hospital and Health Care, Nashville, TN, USA

Clive M. Gray Institute of Infectious Disease and Molecular Medicine, Division of Immunology, Department of Pathology, University of Cape Town, Cape Town, South Africa

Ann F. Green Vanderbilt Institute for Global Health, Vanderbilt University Medical Center, Nashville, TN, USA

Bastian Grewe Department of Molecular and Medical Virology, RuhrUniversity Bochum, Bochum, Germany

Paul Griffiths Centre for Virology, Institute for Immunity and Transplantation, UCL Medical School, London, UK

Andrew E. Grulich HIV Epidemiology and Prevention Program, The Kirby Institute, University of New South Wales, Sydney, NSW, Australia

Adam Grundhoff Heinrich Pette Institute, Leibniz Institute for Experimental Virology, Hamburg, Germany

Jessica Guerra Institute of Human Genetics, Molecular Basis of CancerRelated Inflammation Laboratory, CNRS UPR1142, Montpellier, France

Santiago Guerrero Architecture et Réactivité de l'ARN, UPR 9002 CNRS, Institut de Biologie Moléculaire et Cellulaire du CNRS, Université de Strasbourg, Strasbourg, France

Centre for Genomic Regulation, Barcelona, Spain

Roy M. Gulick Division of Infectious Diseases, Weill Cornell Medicine, New York, NY, USA

Neel K. Gupta Department of Medicine, Division of Hematology/Oncology, University of California, San Francisco, CA, USA

Danielle F. Haley Department of Behavioral Sciences and Health Education, Rollins School of Public Health, Emory University, Atlanta, GA, USA 
H. Irene Hall HIV Incidence and Case Surveillance Branch, Division of HIV/AIDS Prevention, National Center for HIV/AIDS, Viral Hepatitis, STD, and TB Prevention, Centers for Disease Control and Prevention, Atlanta, GA, USA

J. Hampton Atkinson VA San Diego Healthcare System and Department of Psychiatry, University of California, San Diego, San Diego, CA, USA

Rushil Harryparsad Institute of Infectious Disease and Molecular Medicine, Division of Immunology, Department of Pathology, University of Cape Town, Cape Town, South Africa

Christoph Hauser Attending Physician, Department of Infectious Diseases, Bern University Hospital, University of Bern, Bern, Switzerland

Robert K. Heaton Department of Psychiatry, University of California, San Diego, San Diego, CA, USA

Elizabeth Heitman Center for Biomedical Ethics and Society, Vanderbilt University Medical Center, Nashville, TN, USA

Cécile Hérate Inserm U1016, Institut Cochin, Paris, France CNRS, UMR8104, Paris, France

Sorbonne Paris-Cité, Université Paris-Descartes, Paris, France

Diana Hernández Sociomedical Sciences, Columbia University, New York, NY, USA

Carolina Herrera Section of Infectious Diseases, Division of Medicine, Imperial College, London, UK

Mary T. Hickey New York University College of Nursing, New York, NY, USA

Charles Hicks Division of Infectious Diseases, Department of Medicine, University of California San Diego, San Diego, CA, USA

Laurent Hocqueloux CHR d'Orléans- La Source, Service des Maladies Infectieuses et Tropicales, Orléans, France

David R. Holtgrave Department of Health, Behavior and Society, Johns Hopkins Bloomberg School of Public Health, Baltimore, MD, USA

Denise C. Hsu HIV Pathogenesis Unit, Laboratory of Immunoregulation, Division of Intramural Research, National Institute of Allergy and Infectious Diseases, NIH, Bethesda, MD, USA

Lindsey Hutt-Fletcher Department of Microbiology and Immunology, Center for Molecular and Tumor Virology and Feist-Weiller Cancer Center, Louisiana State University Health Sciences Center, Shreveport, LA, USA 
Shiro Ibe Department of Infection and Immunology, Clinical Research Center, National Hospital Organization Nagoya Medical Center, Naka-ku/Nagoya, Aichi, Japan

Andrea Introini Department of Medicine Solna, Karolinska Institute, Center for Molecular Medicine, Karolinska University Hospital, Stockholm, Sweden

Jennifer E. Iudicello Department of Psychiatry, University of California, San Diego, San Diego, CA, USA

Evan S. Jacobs Blood Systems Research Institute, San Francisco, CA, USA

B. Jacquelin Unité de Régulation des Infections Rétrovirales, Institut Pasteur, Paris, Cedex, France

Sabelle Jallow Nuffield Department of Medicine, University of Oxford, Oxford, UK

Marianne Jansson Department of Laboratory Medicine, Division of Medical Microbiology, Lund University, Lund, Sweden

Department of Microbiology, Tumor and Cell Biology (MTC), Karolinska Institute, Stockholm, Sweden

Anthony Jaworowski Department of Infectious Diseases, Alfred Hospital and Monash University, Melbourne, Australia

Centre for Biomedical Research, Burnet Institute, Melbourne, Australia

Department of Immunology, Monash University, Melbourne, Australia

Kuan-Teh Jeang Molecular Virology Section, National Institute of Allergy and Infectious Diseases, Bethesda, MD, USA

Mohammad-Ali Jenabian Département des Sciences Biologiques et Centre de recherche BioMed, Université du Québec à Montréal (UQAM), Montréal, QC, Canada

Mark Y. Jeng Gladstone Institute of Virology and Immunology, University of California, San Francisco, San Francisco, CA, USA

Jeffrey D. Jenks Division of Infectious Diseases, Department of Medicine, The University of California, San Diego, CA, USA

Wim Jennes Department of Biomedical Sciences, Institute of Tropical Medicine, ANR, Belgium

Shibo Jiang Key Laboratory of Medical Molecular Virology of Ministries of Education and Health, Shanghai Medical College and Institute of Medical Microbiology, Fudan University, Shanghai, China

Xiong Jiang Department of Neuroscience, Georgetown University Medical Center, Washington, DC, USA

S. P. Jochems Unité de Régulation des Infections Rétrovirales, Institut Pasteur, Paris, Cedex, France 
Mallory O. Johnson Department of Medicine, Center for AIDS Prevention Studies, University of California, San Francisco, CA, USA

Grace C. John-Stewart Departments of Global Health, Medicine, Epidemiology, and Pediatrics, University of Washington, Seattle, WA, USA

Enid Kabugho Makerere University Johns Hopkins University Research Collaboration, Kampala, Uganda

Phyllis J. Kanki Department of Immunology and Infectious Disease, Harvard School of Public Health, Boston, MA, USA

Shashi Kapadia Division of Infectious Diseases, Department of Medicine, Weill Cornell Medical College, New York, NY, USA

Lawrence D. Kaplan Department of Medicine, Division of Hematology/ Oncology, University of California, San Francisco, CA, USA

Stephen Karpiak ACRIA Center on HIV and Aging, AIDS Community Research Initiative of America (ACRIA) and New York University College of Nursing, New York, NY, USA

Christina Karsten Infection Biology Unit, German Primate Center, Göttingen, Germany

Institute for Cellular Chemistry, Hannover Medical School, Hannover, Germany

Daniel E. Kaufmann The Ragon Institute of MGH, MIT and Harvard, Cambridge, MA, USA

Centre de Recherche du Centre Hospitalier de l'Université de Montréal (CRCHUM) and University of Montreal, Montréal, QC, Canada

Amitinder Kaur Department of Microbiology and Immunology, Tulane University School of Medicine; Tulane National Primate Research Center, Covington, LA, USA

Sheila M. Keating Blood Systems Research Institute, San Francisco, CA, USA

Departments of Laboratory Medicine and Medicine University of California, San Francisco, CA, USA

Sean G. Kelly Division of Infectious Diseases, Department of Medicine, Northwestern University Feinberg School of Medicine, Chicago, IL, USA

Caitlin E. Kennedy Department of International Health, Johns Hopkins Bloomberg School of Public Health, Baltimore, MD, USA

Yoav Kenyan Medical Microbiology Department, University of Manitoba, Winnipeg, MB, Canada

Department of Community Health Sciences, University of Manitoba, Winnipeg, Canada

Department of Internal Medicine, University of Manitoba, Winnipeg, MB, Canada

Department of Medical Microbiology, University of Nairobi, Nairobi, Kenya 
Deanna Kerrigan Department of Health, Behavior and Society, Johns Hopkins Bloomberg School of Public Health, Baltimore, MD, USA

Luc Kestens Department of Biomedical Sciences, Institute of Tropical Medicine, ANR, Belgium

J. Janet Kim Department of Health, Behavior and Society, Johns Hopkins Bloomberg School of Public Health, Baltimore, MD, USA

Jerome H. Kim U.S. Military HIV Research Program, Bethesda, MD, USA

Frank Kirchhoff Institute of Molecular Virology, Ulm University Medical Center, Ulm, Germany

Amy Knowlton Department of Health, Behavior and Society, Johns Hopkins Bloomberg School of Public Health, Baltimore, MD, USA

Dennis L. Kolson Department of Neurology, University of Pennsylvania, Philadelphia, PA, USA

Birgit Korioth-Schmitz Center for Virology and Vaccine Research, Beth Israel Deaconess Medical Center, Harvard Medical School, Boston, MA, USA

Jeffrey E. Korte Department of Public Health Sciences, College of Medicine, Medical University of South Carolina, Charleston, SC, USA

Hans-Georg Kräusslich Department for Infectious Diseases, Virology, University Hospital Heidelberg, Heidelberg, Germany

Susan E. Krown AIDS Malignancy Consortium, New York, NY, USA

Irene Kuo Department of Epidemiology and Biostatistics, George Washington University, Washington, DC, USA

Lillian S. Kuo Virus-Cell Interaction Section, HIV Drug Resistance Program, National Cancer Institute, Frederick, MD, USA

Nadine Laguette Institute of Human Genetics, Molecular Basis of CancerRelated Inflammation Laboratory, CNRS UPR1142, Montpellier, France

Cecile Delille Lahiri Department of Medicine, Division of Infectious Diseases, Emory University School of Medicine, Atlanta, GA, USA

Marie Lambelé Inserm U1016, Institut Cochin, Paris, France

CNRS, UMR8104, Paris, France

Sorbonne Paris-Cité, Université Paris-Descartes, Paris, France

Cameron Lambert Emory University School of Medicine, Atlanta, GA, USA

Olivier Lambotte Department of Clinical Immunology, Paris South University Hospitals, Le Kremlin Bicêtre, France

Alan Landay Affymetrix, San Diego, CA, USA

Carl A. Latkin Department of Health, Behavior and Society, Johns Hopkins Bloomberg School of Public Health, Baltimore, MD, USA 
Anh Q. Le Faculty of Health Sciences, Simon Fraser University, Burnaby, $\mathrm{BC}$, Canada

Sylvie Le Gall Ragon Institute of MGH, MIT and Harvard, Massachusetts General Hospital, Harvard Medical School, Cambridge, MA, USA

Stuart F. J. Le Grice RT Biochemistry Section, Basic Research Laboratroy, Frederick National Laboratory for Cancer Research, National Cancer Institute, Frederick, MD, USA

Jasper S. Lee Department of Psychology, University of Miami, Coral Gables, FL, USA

Bethany Lema Department of Dermatology, Roswell Park Cancer Institute, Buffalo, NY, USA

Philippe Lemey Department of Microbiology and Immunology, Rega Institute for Medical Research, Leuven, KU, Belgium

Jeffrey Lennox Department of Medicine, Division of Infectious Diseases, Emory University School of Medicine, Atlanta, GA, USA

Andrew M. L. Lever University of Cambridge, Cambridge, UK

Andrew J. Levine Department of Neurology, David Geffen School of Medicine at the University of California, Los Angeles, CA, USA

Sharon R. Lewin Department of Infectious Diseases, Alfred Hospital and Monash University, Melbourne, VIC, Australia

Centre for Biomedical Research, Burnet Institute, Melbourne, VIC, Australia

Crystal Fuller Lewis Division of Social Solutions and Services Research, Nathan Kline Institute for Psychiatric Research, New York State Office of Mental Health, NY, USA

George K. Lewis Division of Basic Science and Vaccine Research, Institute of Human Virology, University of Maryland School of Medicine, Baltimore, MD, USA

Rashunda Lewis Capstone College of Nursing, The University of Alabama, Tuscaloosa, AL, USA

Qingsheng Li School of Biological Sciences and Nebraska Center for Virology, University of Nebraska-Lincoln, Lincoln, NE, USA

Frank Liang Department of Medicine, Clinical Immunology and Allergy Unit, Karolinska Institutet and University Hospital, Stockholm, Sweden

Camille Libre Architecture et Réactivité de l'ARN, UPR 9002 CNRS, Institut de Biologie Moléculaire et Cellulaire du CNRS, Université de Strasbourg, Strasbourg, France

Mathias Lichterfeld Infectious Disease Division, Brigham and Women's Hospital, Boston, MA, USA 
Mathias Lichterfeld Ragon Institute of MGH, MIT and Harvard, Boston, MA, USA

Infectious Disease Division, Massachusetts General Hospital, Boston, MA, USA

Rupali J. Limaye Department of International Health, Johns Hopkins Bloomberg School of Public Health, Baltimore, MD, USA

Zhiyi Lin Department of Pharmaceutical Sciences, University of Nebraska Medical Center, Omaha, NE, USA

Department of Pharmacology and Experimental Neuroscience, University of Nebraska Medical Center, Omaha, NE, USA

Mary Lou Lindegren Department of Pediatrics and the Department of Health Policy, Vanderbilt Institute for Global Health, Nashville, TN, USA

Madelene Lindqvist Ragon Institute of MGH, MIT and Harvard, Cambridge, MA, USA

Anna Lissina Centre d'Immunologie et des Maladies Infectieuses (CIMIParis), Sorbonne Universités, UPMC Univ Paris 06, CR7, Paris, France

INSERM, U1135, CIMI-Paris, Paris, France

Richard F. Little Clinical Investigations Branch, Cancer Therapy Evaluation Program, Division of Cancer Treatment and Diagnosis (DCTD), National Cancer Institute (NCI), Bethesda, MD, USA

Yu Liu Vanderbilt Institute for Global Health, Vanderbilt University, Nashville, TN, USA

Shawnta L. Lloyd Department of Epidemiology, Rollins School of Public Health, Emory University, Atlanta, GA, USA

Andrew Lloyd Inflammation and Infection Research Centre, University of New South Wales, Sydney, Australia

Prince of Wales Clinical School, University of New South Wales, Randwick, Australia

Karin Loré Department of Medicine, Clinical Immunology and Allergy Unit, Karolinska Institutet and University Hospital, Stockholm, Sweden

Sana Loue Department of Bioethics, School of Medicine, Case Western Reserve University, Cleveland, OH, USA

Lu Lu Key Laboratory of Medical Molecular Virology of Ministries of Education and Health, Shanghai Medical College and Institute of Medical Microbiology, Fudan University, Shanghai, China

Jeremy Luban Program in Molecular Medicine, Department of Biochemistry and Molecular Pharmacology, University of Massachusetts Medical School, Worcester, MA, USA

Irene Lubega Makerere University Johns Hopkins University Research Collaboration, Kampala, Uganda 
Bojana Lucic Department of Infectious Diseases, Integrative Virology, University Hospital Heidelberg and German Centre for Infection Research, Heidelberg, Germany

Jacob G. Ludington Sackler School of Graduate Biomedical Sciences and Tufts Medical Center, Tufts University, Boston, MA, USA

Marina Lusic Department of Infectious Diseases, Integrative Virology, University Hospital Heidelberg and German Centre for Infection Research, Heidelberg, Germany

Katherine Luzuriaga Program in Molecular Medicine, University of Massachusetts Medical School, Worcester, MA, USA

Department of Pediatrics, University of Massachusetts Medical School, Worcester, MA, USA

Cynthia M. Lyles Division of HIV/AIDS Prevention, The U.S. Centers for Disease Control and Prevention, Atlanta, GA, USA

Jennifer L. Lyons Department of Neurology, Division of Neurological Infections and Inflammatory Diseases, Brigham and Women's Hospital and Harvard Medical School, Boston, MA, USA

Jessica MacFarlane Duke Global Health Institute, Duke University, Durham, NC, USA

Keletso Makofane Global Forum on MSM and HIV (MSMGF), Oakland, CA, USA

Shokouh Makvandi-Nejad Radcliffe Department of Medicine, University of Oxford, Oxford, UK

Robert Malow Department of Health Promotion and Disease Prevention, Robert Stempel College of Public Health and Social Work, Florida International University, Miami, FL, USA

Deepthi Mani Division of Internal Medicine, Multicare Good Samaritan Hospital, Puyallup, WA, USA

Kamal Mansinho Serviço de Infecciologia e Medicina Tropical, Hospital de Egas Moniz, Centro Hospitalar de Lisboa Ocidental, E.P.E., Lisbon, Portugal

Fredrik Månsson Department of Clinical Sciences Malmö, Lund University, Malmö, Sweden

Andrea Mantsios Department of Health, Behavior and Society, Johns Hopkins Bloomberg School of Public Health, Baltimore, MD, USA

Christian Manzardo Infectious Diseases Service, Hospital Clínic-IDIBAPS, University of Barcelona, Barcelona, Spain 
Ben Marais The Children's Hospital at Westmead and the Marie Bashir Institute for Infectious Diseases and Biosecurity, The University of Sydney, Syndey, Australia

Anne-Geneviève Marcelin Sorbonne Universités, UPMC Univ Paris 06, INSERM, Institut Pierre Louis d'épidémiologie et de Santé Publique (IPLESP UMRS 1136), Paris, France

Laboratoire de Virologie, AP-HP, Groupe hospitalier Pitié Salpêtrière, Paris, France

Alessandro Marcello Laboratory of Molecular Virology, International Centre for Genetic Engineering and Biotechnology (ICGEB), AREA Science Park, Trieste, Italy

Vincent C. Marconi Division of Infectious Diseases, Emory University School of Medicine, Atlanta, GA, USA

Daniel F. Marker Center for Neural Development and Disease, Department of Neurology, University of Rochester Medical Center, Rochester, NY, USA

Roland Marquet Architecture et Réactivité de l'ARN, UPR 9002 CNRS, Institut de Biologie Moléculaire et Cellulaire du CNRS, Université de Strasbourg, Strasbourg, France

Maureen P. Martin Cancer and Inflammation Program, Laboratory of Experimental Immunology, Leidos Biomedical Research Inc., Frederick National Laboratory for Cancer Research, Frederick, MD, USA

Ragon Institute of MGH, MIT and Harvard, Boston, MA, USA

Eileen Martin Department of Psychiatry, Rush University Medical Center, Chicago, IL, USA

Elena Martinelli Center for Biomedical Research, Population Council, New York, NY, USA

Jose M. Martinez-Navio Department of Pathology, University of Miami Miller School of Medicine, Miami, FL, USA

Sophie Matheron Service de Maladies Infectieuses et Tropicales, BichatClaude Bernard Hospital and University, Paris, France

Cathy Maulsby Department of Health, Behavior and Society, Johns Hopkins Bloomberg School of Public Health, Baltimore, MD, USA

Kenneth H. Mayer Fenway Health, The Fenway Institute, Boston, MA, USA

Division of Infectious Diseases, Department of Medicine, Beth Israel Deaconess Medical Center, Harvard Medical School, Boston, MA, USA

Souleymane Mboup Department of Virologie, Cheikh Anta Diop Université, Dakar, Senegal 
Sam Mbulaiteye Infections and Immunoepidemiology Branch, National Cancer Institute, Bethesda, MD, USA

Sam M. Mbulaiteye Infections and Immunoepidemiology Branch, Division of Cancer Epidemiology and Genetics, Department of Health and Human Services, National Cancer Institute, National Institutes of Health, Bethesda, MD, USA

Michael B. McChesney California National Primate Research Center, University of California, Davis, CA, USA

Allison McFall Department of Epidemiology, Johns Hopkins Bloomberg School of Public Health, Baltimore, MD, USA

Colleen S. McGary Division of Microbiology and Immunology, Yerkes National Primate Research Center, Atlanta, GA, USA

Lyle R. McKinnon Mucosal Immunology Laboratory, Centre for the AIDS Programme of Research in South Africa (CAPRISA), Durban, South Africa

Paul McLaren School of Life Sciences, Ecole Polytechnique Fédérale de Lausanne, Lausanne, Vaud, Switzerland

Junette McWilliams Department of Health, Behavior and Society, Johns Hopkins Bloomberg School of Public Health, Baltimore, MD, USA

Patrik Medstrand Department of Laboratory Medicine Malmö, Lund University, Malmö, Sweden

Rick B. Meeker Department of Neurology, University of North Carolina School of Medicine, Chapel Hill, NC, USA

Graeme Meintjes Institute of Infectious Diseases and Molecular Medicine and Department of Medicine, University of Cape Town, Cape Town, South Africa

Lauren C. Messina Department of Epidemiology, Rollins School of Public Health, Emory University, Atlanta, GA, USA

Lisa R. Metsch Department of Sociomedical Sciences, Mailman School of Public Health, Columbia University, New York, NY, USA

Nelson L. Michael U.S. Military HIV Research Program, Bethesda, MD, USA

Christopher J. Miller Center for Comparative Medicine; and Department of Pathology, Microbiology and Immunology, School of Veterinary Medicine; and Division of Infectious Diseases, Department of Internal Medicine, School of Medicine; and California National Primate Research Center, University of California, Davis, CA, USA

Jennifer T. Miller RT Biochemistry Section, Basic Research Laboratroy, Frederick National Laboratory for Cancer Research, National Cancer Institute, Frederick, MD, USA 
Caitlin Milligan Fred Hutchinson Cancer Research Center, Seattle, WA, USA

Matthew J. Mimiaga Brown University School of Public Health, Providence, RI, USA

Brown University Alpert Medical School, Providence, RI, USA

Brown University Institute for Community Health Promotion, Providence, RI, USA

Harvard T.H. Chan School of Public Health, Boston, MA, USA

The Fenway Institute, Fenway Health, Boston, MA, USA

José M. Miró Infectious Diseases Service, Hospital Clínic-IDIBAPS, University of Barcelona, Barcelona, Spain

Ana Cláudia Miranda Serviço de Infecciologia e Medicina Tropical, Hospital de Egas Moniz, Centro Hospitalar de Lisboa Ocidental, E.P.E., Lisbon, Portugal

Ronald T. Mitsuyasu UCLA Center for Clinical AIDS Research and Education (CARE Center), University of California, Los Angeles, Los Angeles, CA, USA

Jennifer A. Mitty Beth Israel Deaconess Medical Center, Harvard Medical School, Boston, MA, USA

Brown University Alpert Medical School, Providence, RI, USA

Abeer Moanna Emory University School of Medicine, Atlanta, GA, USA

Susan Moir Laboratory of Immunoregulation, National Institute of Allergy and Infectious Diseases, National Institutes of Health, Besthesda, MD, USA

Yamile Molina Public Health Sciences Division, Fred Hutchinson Cancer Research Center, Seattle, WA, USA

Brooke E. E. Montgomery Fay W. Boozman College of Public Health, University of Arkansas for Medical Sciences, Little Rock, AR, USA

Mary W. Montgomery Infectious Diseases Division, Brigham and Women's Hospital, Harvard Medical School, Boston, MA, USA

Jessica L. Montoya SDSU/UCSD Joint Doctoral Program in Clinical Psychology, San Diego State University/University of California, San Diego, CA, USA

Christiane Moog Vaccine Research Institute (VRI), Créteil, France

INSERM U1109, Institut de Virologie, Fédération de Médecine Translationnelle de Strasbourg (FMTS), Faculté de Médecine, Université de Strasbourg, Strasbourg, France

Troy D. Moon Vanderbilt Institute for Global Health, Department of Pediatrics, Division of Infectious Diseases, Vanderbilt University Medical Center, Nashville, TN, USA 
David J. Moore Department of Psychiatry, University of California, San Diego, UCSD HNRC, San Diego, CA, USA

Maria E. Moreno-Fernandez Department of Immunobiology, Cincinnati Children Hospital Medical Center, Cincinnati, OH, USA

Ruth Morgan Thomas Global Network of Sex Work Projects (NSWP), Edinburgh, Scotland, UK

Charles Muiruri Duke Global Health Institute, Durham, NC, USA

Barbara Müller Department for Infectious Diseases, Virology, University Hospital Heidelberg, Heidelberg, Germany

M. Müller-Trutwin Unité de Régulation des Infections Rétrovirales, Institut Pasteur, Paris, France

Shannon Murray Vaccine and Gene Therapy Institute of Florida, Port St Lucie, FL, USA

Peninah Mwangi Bar Hostess Empowerment and Support Program, Nairobi, Kenya

Athi Myint-U Education Development Center, Inc., Waltham, MA, USA

Kimberly M. Nelson Department of Psychology, University of Washington, Seattle, WA, USA

David Nicolás Infectious Diseases Service, Hospital Clínic-IDIBAPS, University of Barcelona, Barcelona, Spain

Susanne Dam Nielsen Department of Infectious Diseases, University of Copenhagen, Rigshospitalet, Copenhagen, Denmark

Sébastien Nisole INSERM UMR-S 1124, Université Paris Descartes, Paris, France

Sandisiwe Noholoza Institute of Infectious Disease and Molecular Medicine, Division of Immunology, Department of Pathology, University of Cape Town, Cape Town, South Africa

Philip J. Norris Blood Systems Research Institute, San Francisco, CA, USA Departments of Laboratory Medicine and University of California, San Francisco, CA, USA

Rebecca G. Nowak Division of Epidemiology and Prevention, Institute of Human Virology, University of Maryland School of Medicine, Baltimore, USA

Salma Nowroozalizadeh Department of Laboratory Medicine, Division of Medical Microbiology, Lund University, Lund, Sweden

Samuel Victor Nuvor School of Medical Sciences, University of Cape Coast, Cape Coast, Ghana, West Africa

Lydia O'Donnell Education Development Center, Inc., Waltham, MA, USA 
Ighovwerha Ofotokun Department of Medicine, Division of Infectious Diseases, Emory University School of Medicine, Atlanta, GA, USA

Bibilola D. Oladeji Department of Neurology, University of North Carolina School of Medicine, Chapel Hill, NC, USA

Department of Psychiatry, College of Medicine University of Ibadan, Ibadan, Nigeria

Abraham J. Olivier Institute of Infectious Disease and Molecular Medicine, Division of Immunology, Department of Pathology, University of Cape Town, Cape Town, South Africa

Habib R. Omar Kilimanjaro Christian Medical Center, Moshi, Tanzania

Alexandra M. Ortiz Laboratory of Molecular Microbiology, NIAID, National Institutes of Health, Bethesda, MD, USA

Mario Ostrowski Department of Medicine, University of Toronto, Toronto, ON, Canada

Keenan Research Centre for Biomedical Science of St. Michael's Hospital, Toronto, ON, Canada

Melanie Ott Gladstone Institute of Virology and Immunology, University of California San Francisco, San Francisco, CA, USA

Julie Overbaugh Fred Hutchinson Cancer Research Center, Seattle, WA, USA

Stefan Pöhlmann Infection Biology Unit, German Primate Center, Göttingen, Germany

Mirko Paiardini Division of Microbiology and Immunology, Yerkes National Primate Research Center, Atlanta, GA, USA

Jean-Christophe Paillart Architecture et Réactivité de l'ARN, UPR 9002 CNRS, Institut de Biologie Moléculaire et Cellulaire du CNRS, Université de Strasbourg, Strasbourg, France

Joel Palefsky Department of Infectious Diseases, University of California, San Francisco, CA, USA

Gianfranco Pancino Institut Pasteur and INSERM, Paris, France

Ivona Pandrea Center for Vaccine Research and Department of Pathology, School of Medicine, University of Pittsburgh, Pittsburgh, PA, USA

Laure Papin Centre d'études d'agents Pathogènes et Biotechnologies pour la Santé (CPBS), CNRS-UMR, Montpellier, France

Carrigan Parish Department of Sociomedical Sciences, Mailman School of Public Health, Miami Research Center, Columbia University, Miami, FL, USA

Department of Public Health Sciences, Miller School of Medicine, University of Miami, Miami, FL, USA 
Jo-Ann S. Passmore Mucosal Immunology Laboratory, Centre for the AIDS Programme of Research in South Africa (CAPRISA), Durban, South Africa Institute of Infectious Diseases and Molecular Medicine (IDM), University of Cape Town, Cape Town, South Africa

National Health Laboratory Services, Cape Town, South Africa

C. David Pauza Department of Medicine, Institute of Human Virology, University of Maryland School of Medicine, Baltimore, MD, USA

William A. Paxton Department of Clinical Infection, Microbiology and Immunology, Institute of Infection and Global Health, University of Liverpool, Liverpool, UK

K. Michelle Peavy Alcohol and Drug Abuse Institute, University of Washington, Seattle, WA, USA

Evergreen Treatment Services, Seattle, WA, USA

Maryse Peressin INSERM U1110, Université de Strasbourg, Strasbourg, France

Amaya Perez-Brumer The Fenway Institute, Fenway Health, Boston, MA, USA

Columbia University Mailman School of Public Health, New York, NY, USA

Kevin Peterson Department of Clinical Sciences, Institute of Tropical Medicine, Antwerp, Belgium

Department of Epidemiology and Social Medicine, Faculty of Medical Sciences, University of Antwerp, Antwerp, Belgium

Gregory Phillips II Department of Medical Social Sciences, Feinberg School of Medicine, Northwestern University, Chicago, IL, USA

Stefania Pittaluga Laboratory of Pathology, Center for Cancer Research (CCR), Bethesda, MD, USA

M. J. Ploquin Unité de Régulation des Infections Rétrovirales, Institut Pasteur, Paris, France

Anthony T. Podany Antiviral Pharmacology Laboratory and Department of Pharmacy Practice, College of Pharmacy, University of Nebraska Medical Center, Omaha, NE, USA

Mark N. Polizzotto HIV and AIDS Malignancy Branch, National Cancer Institute, Bethesda, MD, USA

Georgios Pollakis Department of Clinical Infection, Microbiology and Immunology, Institute of Infection and Global Health, University of Liverpool, Liverpool, UK

Filippos Porichis The Ragon Institute of MGH, MIT and Harvard, Cambridge, MA, USA

Owen Pornillos Department of Molecular Physiology and Biological Physics, University of Virginia, Charlottesville, VA, USA 
Christopher Power Department of Medicine (Neurology), University of Alberta, Edmonton, AB, Canada

Guillermo Prado Department of Public Health Sciences, University of Miami, Miami, FL, USA

Richard W. Price Department of Neurology, UCSF School of Medicine, San Francisco General Hospital, San Francisco, CA, USA

Eve Puffer Department of Psychology and Neuroscience, Duke University, Durham, NC, USA

Duke Global Health Institute, Duke University, Durham, NC, USA

Han-Zhu Qian Division of Epidemiology, Vanderbilt University Medical Center, Vanderbilt Institute for Global Health, Nashville, TN, USA

The Centers for Disease Control and Prevention, Atlanta, GA, USA

Lisa Rahangdale Department of Obstetrics and Gynecology, University of North Carolina at Chapel Hill, Chapel Hill, NC, USA

Nadia Rahm Unité Propre de Recherche 1142, Laboratoire de Virologie Moléculaire, Institut de Génétique Humaine, Centre National de la Recherche Scientifique, Montpellier, France

Radha Rajasingham Division of Infectious Diseases and International Medicine, Department of Medicine, University of Minnesota, Minneapolis, MN, USA

Isobel Ramsay Centre for Virology, Institute for Immunity and Transplantation, UCL Medical School, London, UK

Srinika Ranasinghe Ragon Institute of MGH, MIT and Harvard, Cambridge, MA, USA

Shreya Rao Hubert Department of Global Health, Rollins School of Public Health, Emory University, Atlanta, GA, USA

Emory University School of Medicine, Atlanta, GA, USA

Andri Rauch Department of Infectious Diseases, Inselspital, Bern University Hospital, University of Bern, Bern, Switzerland

Sindhu Ravishankar Substance Use, Gender and Applied Research, RTI International, Research Triangle Park, NC, USA

Erin Reid Department of Internal Medicine, University of California, San Diego Moores Cancer Center, La Jolla, CA, USA

Nancy R. Reynolds Yale University, West Haven, CT, USA

Sushena Reza-Paul University of Manitoba, Winnipeg, MB, Canada

Jan De Rijck Laboratory for Molecular Virology and Gene Therapy, KU Leuven, Leuven, Flanders, Belgium

Stacey A. Rizza Division of Infectious Diseases, Mayo Clinic, Rochester, MN, USA 
Merlin L. Robb U.S. Military HIV Research Program, Bethesda, MD, USA

Melissa Robbiani Center for Biomedical Research, Population Council, New York, NY, USA

Erle S. Robertson Department of Microbiology, Perelman School of Medicine, University of Pennsylvania, Philadelphia, PA, USA

Kevin Robertson Department of Neurology, University of North Carolina School of Medicine, Chapel Hill, NC, USA

Jürgen Kurt Rockstroh Department of Medicine, University Hospital Bonn, Bonn, Germany

Allan E. Rodriguez University of Miami, Miller School of Medicine, Miami, FL, USA

Brooke G. Rogers Department of Psychology, University of Miami, Coral Gables, FL, USA

Thomas J. Rogers Center for Inflammation, Translational and Clinical Lung Research, Temple University School of Medicine, Philadelphia, PA, USA

Keshet Ronen Fred Hutchinson Cancer Research Center, Seattle, WA, USA

Pierre Roques Service d'Immunovirologie, Institute of Emerging Disease and Innovative Therapy, Commissariat à l'Energie Atomique, Fontenay-auxRoses, France

UMR-E1 Université Paris Sud-XI, Orsay, France

Retrovirology Department, CIRMF, Franceville, Gabon Year 2001-2004

Michael Rosenberg Division of Pediatric Infectious Diseases, Jacobi Medical Center, Albert Einstein College of Medicine, Yeshiva University, Bronx, NY, USA

J. Megan Ross Department of Psychology, Florida International University, Miami, FL, USA

Christine Rouzioux Hopital Necker, Laboratoire de Virologie, Université Paris Descartes, Paris, France

Université Paris Descartes, Paris, France

Sarah L. Rowland-Jones Nuffield Department of Medicine, NDM Research Building, University of Oxford, Headington, Oxford, UK

Marijana Rucevic Ragon Institute of MGH, MIT and Harvard, Harvard Medical School, Cambridge, MA, USA

Cesar M. Rueda Department of Immunobiology, Cincinnati Children Hospital Medical Center, Cincinnati, OH, USA

Richard M. Rutstein Division of General Pediatrics, Children's Hospital of Philadelphia, Philadelphia, PA, USA

Asier Sáez-Cirión Institut Pasteur, Unité de Régulation des Infections Rétrovirales, Paris, France 
Steven A. Safren Department of Psychology, University of Miami, Coral Gables, FL, USA

Amy M. Sainski Department of Molecular Pharmacology and Experiment Therapeutics, Mayo Clinic, Rochester, MN, USA

Theo Sandfort HIV Center for Clinical and Behavioral Studies, New York State Psychiatric Institute and Columbia University, New York, NY, USA

Kerrie J. Sandgren Centre for Virus Research, Westmead Millennium Institute, Sydney, Australia

Department of Medicine, Clinical Immunology and Allergy Unit, Karolinska Institutet and University Hospital, Stockholm, Sweden

Mario L. Santiago Department of Medicine, Division of Infectious Diseases, University of Colorado Anschutz Medical Campus, Aurora, CO, USA

Delphine Sauce Centre d'Immunologie et des Maladies Infectieuses (CIMIParis), Sorbonne Universités, UPMC Univ Paris 06, CR7, Paris, France

INSERM, U1135, CIMI-Paris, Paris, France

Daniel Sauter Institute of Molecular Virology, Ulm University Medical Center, Ulm, Germany

Paul E. Sax Infectious Diseases Division, Brigham and Women's Hospital, Harvard Medical School, Boston, MA, USA

Gabriella Scarlatti Viral Evolution and Transmission Unit, Division of Immunology, Transplantation and Infectious Diseases, San Raffaele Scientific Institute, Milan, Italy

Jörn E. Schmitz Center for Virology and Vaccine Research, Beth Israel Deaconess Medical Center, Harvard Medical School, Boston, MA, USA

Rik Schrijvers Laboratory for Molecular Virology and Gene Therapy, KU Leuven, Leuven, Flanders, Belgium

Eric W. Schrimshaw Sociomedical Sciences, Mailman School of Public Health, Columbia University, New York, NY, USA

David Sears Division of HIV Medicine, Department of Medicine, HarborUCLA Medical Center, David Geffen School of Medicine at UCLA, Torrance, CA, USA

Soraya Seedat Faculty of Medicine and Health Sciences, Department of Psychiatry, University of Stellenbosch, Cape Town, Western Cape, South Africa

Katherine Semrau Center for Global Health and Development, Boston University of Public Health, Boston, MA, USA

Irini Sereti HIV Pathogenesis Unit, Laboratory of Immunoregulation, Division of Intramural Research, National Institute of Allergy and Infectious Diseases, NIH, Bethesda, MD, USA 
Paola Sette Laboratory of Molecular Microbiology, National Institute of Allergy and Infectious Diseases, National Institutes of Health, Bethesda, MD, USA

Jae Sevelius Department of Medicine, Center for AIDS Prevention Studies, Center of Excellence for Transgender Health, University of California, San Francisco, CA, USA

Barbara L. Shacklett Department of Medical Microbiology and Immunology, School of Medicine, University of California, Davis, Davis, CA, USA

Vaibhav B. Shah Department of Pathology, Microbiology and Immunology, Vanderbilt University School of Medicine, Nashville, TN, USA

Aniqa Shahid Faculty of Health Sciences, Simon Fraser University, Burnaby, BC, Canada

Kate Shannon Gender and Sexual Health Initiative, BC Centre for Excellence in HIV/AIDS, St. Paul's Hospital, Vancouver, BC, Canada

Division of AIDS, Department of Medicine, Faculty of Medicine, University of British Columbia, Vancouver, BC, Canada

School of Population and Public Health, Faculty of Medicine, University of British Columbia, Vancouver, BC, Canada

Robin Shattock Section of Infectious Diseases, Division of Medicine, Imperial College, London, UK

Stacey A. Shaw Social Intervention Group, Columbia University School of Social Work, NY, USA

Susan G. Sherman Department of Health, Behavior and Society, Department of Epidemiology, Johns Hopkins Bloomberg School of Public Health, Baltimore, MD, USA

Maria Aparecida Shikanai-Yasuda Department of Infectious and Parasitic Diseases, Faculdade de Medicina, Laboratory of Clinical Investigation on Immunology, Hospital das Clínicas, University of São Paulo, Sao Paulo, SP, Brazil

Basile Siewe Affymetrix, San Diego, CA, USA

Kathleen Sikkema Department of Psychology and Neuroscience, Duke University, Durham, NC, USA

Duke Global Health Institute, Duke University, Durham, NC, USA

Kenneth O. Simbiri Department of Microbiology, Perelman School of Medicine, University of Pennsylvania, Philadelphia, PA, USA

Jane M. Simoni Department of Psychology, University of Washington, Seattle, WA, USA

Shmona Simpson Nuffield Department of Medicine, University of Oxford, Oxford, UK 
Harjot K. Singh Division of Infectious Diseases, Department of Medicine, Weill Cornell Medical College, New York, NY, USA

Anna Smed-Sörensen Department of Medicine, Clinical Immunology and Allergy Unit, Karolinska Institutet and University Hospital, Stockholm, Sweden

Erin Smith Lydia O’Donnell, Ed.D., Education Development Center, Inc., Waltham, MA, USA

Renee Smith Department of Pediatrics, University of Illinois at Chicago, Chicago, USA

Mohan Somasundaran Department of Pediatrics, University of Massachusetts Medical School, Worcester, MA, USA

James L. Sorensen Department of Psychiatry, University of California, San Francisco, CA, USA

Cathia Soulie Sorbonne Universités, UPMC Univ Paris 06, INSERM, Institut Pierre Louis d'épidémiologie et de Santé Publique (IPLESP UMRS 1136), Paris, France

Laboratoire de Virologie, AP-HP, Groupe hospitalier Pitié Salpêtrière, Paris, France

Ana E. Sousa Unidade de Imunologia Clínica, Instituto de Medicina Molecular, Faculdade de Medicina, Universidade de Lisboa, Lisbon, Portugal

Anne C. Spaulding Department of Epidemiology, Rollins School of Public Health, Emory University, Atlanta, GA, USA

Mark Spear Department of Molecular and Microbiology, National Center for Biodefense and Infectious Diseases, George Mason University, Manassas, VA, USA

Michele Spina Division of Medical Oncology A, National Cancer Institute, Aviano, PN, Italy

Corine St. Gelais Department of Veterinary Biosciences, Center for Retrovirus Research, The Ohio State University, Columbus, OH, USA

Shauna Stahlman Johns Hopkins Bloomberg School of Public Health, Center for Public Health and Human Rights, Department of Epidemiology, Baltimore, MD, USA

D. Cristina Stefan Department of Paediatrics and Child Health, Tygerberg Hospital and Stellenbosch University, Tygerberg, Cape Town, South Africa

Katharine E. Stewart Academic Affairs and Learning Strategies, University of North Carolina General Administration, Chapel Hill, NC, USA

Elizabeth A. Stier Obstetrics and Gynecology, Boston University Medical Center, Boston, MA, USA

Steffanie A. Strathdee Gender and Sexual Health Initiative, BC Centre for Excellence in HIV/AIDS, St. Paul's Hospital, Vancouver, BC, Canada

Division of Global Public Health in the Department of Medicine, San Diego School of Medicine, University of California, San Diego, USA 
Erich M. Sturgis Department of Head and Neck Surgery and Department of Epidemiology, The University of Texas MD Anderson Cancer Center, Houston, TX, USA

Bin Su Vaccine Research Institute (VRI), Créteil, France

INSERM U1109, Institut de Virologie, Fédération de Médecine Translationnelle de Strasbourg (FMTS), Faculté de Médecine, Université de Strasbourg, Strasbourg, France

Beijing Key Laboratory for HIV/AIDS Research, Center for Infectious Diseases, Beijing You'an Hospital, Capital Medical University, Beijing, China

Sujit Suchindran Divisions of Infectious Diseases and General Medicine, Massachusetts General Hospital, Boston, MA, USA

Wataru Sugiura Department of Infection and Immunology, Clinical Research Center, National Hospital Organization Nagoya Medical Center, Naka-ku/Nagoya, Aichi, Japan

Department of AIDS Research, Nagoya University Graduate School of Medicine, Nagoya, Aichi, Japan

Patrick S. Sullivan Department of Epidemiology, Rollins School of Public Health, Emory University, Atlanta, USA

Khuanchai Supparatpinyo Division of Infectious Diseases, Faculty of Medicine, Chiang Mai University, Chiang Mai, Thailand

Research Institute for Health Sciences, Chiang Mai University, Chiang Mai, Thailand

Richard E. Sutton Section of Infectious Diseases, Yale University, NewHaven, CT, USA

Ronald Swanstrom The University of North Carolina, Lineberger Cancer Center, Chapel Hill, NC, USA

Michael D. Sweat Department of Psychiatry and Behavioral Sciences, Division of Global and Community Health, Medical University of South Carolina, Charleston, SC, USA

Babafemi O. Taiwo Division of Infectious Diseases, Northwestern University Feinberg School of Medicine, Chicago, IL, USA

Oliver Taltynov Laboratory for Molecular Virology and Gene Therapy, KU Leuven, Leuven, Flanders, Belgium

Karen T. Tashima The Miriam Hospital, Providence, RI, USA

Department of Medicine, The Warren Alpert Medical School of Brown University, Providence, RI, USA

Nuno Taveira Centro de Investigação Interdisciplinar Egas Moniz (CiiEM), Instituto Superior de Ciências da Saúde Egas Moniz (ISCSEM), Monte de Caparica, and Research Institute for Medicines (iMed.ULisboa)/Unidade dos Retrovírus e Infecções Associadas (URIA), Centro de Patogénese Molecular, Faculty of Pharmacy, University of Lisbon, Lisbon, Portugal 
Tonya N. Taylor College of Medicine/Special Treatment and Research (STAR) Program, SUNY Downstate Medical Center, Brooklyn, NY, USA

Philip R. Tedbury HIV Drug Resistance Program, Frederick National Laboratory, Frederick, MD, USA

Amalio Telenti The J Craig Venter Institute, La Jolla, CA, USA

Rita Tendeiro Unidade de Imunologia Clínica, Instituto de Medicina Molecular, Faculdade de Medicina, Universidade de Lisboa, Lisbon, Portugal

Kiki Tesselaar Laboratory of Translational Immunology, Department of Immunology, University Medical Center Utrecht, Utrecht, The Netherlands

Umberto Tirelli Division of Medical Oncology A, National Cancer Institute, Aviano, PN, Italy

Nagaraja Tirumuru Department of Veterinary Biosciences, Center for Retrovirus Research, The Ohio State University, Columbus, OH, USA

Lauren Tompkins The University of North Carolina, Lineberger Cancer Center, Chapel Hill, NC, USA

Giovanna Tosato Laboratory of Cellular Oncology, Center for Cancer Research, National Cancer Institute, Bethesda, MD, USA

Sima S. Toussi Division of Pediatric Infectious Diseases, Department of Pediatrics, NY Presbyterian Hospital - Weill Cornell Medical College, New York, NY, USA

Francine Touzard Romo The Miriam Hospital, Providence, RI, USA

Department of Medicine, The Warren Alpert Medical School of Brown University, Providence, RI, USA

Lydie Trautmann Vaccine and Gene Therapy Institute of Florida, Port Saint Lucie, FL, USA

Virginia A. Triant Divisions of Infectious Diseases and General Medicine, Massachusetts General Hospital, Boston, MA, USA

Susan Tross HIV Center for Clinical and Behavioral Studies, New York State Psychiatric Institute, Columbia University Medical Center, New York, NY, USA

Klaus Überla Department of Molecular and Medical Virology, RuhrUniversity Bochum, Bochum, Germany

Thomas S. Uldrick Retroviral Disease Section, HIV and AIDS Malignancy Branch, Center for Cancer Research, National Cancer Institute (NCI), Bethesda, MD, USA

Anneli Uuskula Department of Family Medicine and Public Health, University of Tartu, Tartu, Estonia 
B. Van Driessche Institute for Molecular Biology and Medicine (IBMM), Université Libre de Bruxelles (ULB), Service of Molecular Virology, Gosselies, Belgium

C. Van Lint Institute for Molecular Biology and Medicine (IBMM), Université Libre de Bruxelles (ULB), Service of Molecular Virology, Gosselies, Belgium

Anne-Mieke Vandamme Global Health and Tropical Medicine, Institute for Hygiene and Tropical Medicine, Universidade Nova de Lisboa, Lisbon, Portugal

Department of Microbiology and Immunology, Rega Institute for Medical Research, Clinical and Epidemiological Virology, KU Leuven-University of Leuven, Leuven, Belgium

Unit for Microbiology, Institute for Hygiene and Tropical Medicine, Universidade Nova de Lisboa, Lisbon, Portugal

Janneke van de Wijgert Department of Clinical Infection, Microbiology and Immunology, Institute of Infection and Global Health, University of Liverpool, Liverpool, UK

Leigh van den Heuvel Faculty of Medicine and Health Sciences, Department of Psychiatry, University of Stellenbosch, Cape Town, Western Cape, South Africa

Maarten Schim van der Loeff Department of Infectious Diseases, Public Health Service of Amsterdam (GGD), Amsterdam, The Netherlands

Center for Infection and Immunity Amsterdam (CINIMA), Department of Internal Medicine, Academic Medical Center, Amsterdam, The Netherlands

Jan van Lunzen Infectious Diseases Unit, University Medical Center Eppendorf and Heinrich Pette Institute - Leibniz Institute for Experimental Virology, Hamburg, Germany

Carla van Tienen Department of Medical Microbiology and Infectious Diseases, Erasmus Medical Centre, Rotterdam, The Netherlands

Peter Veldkamp Department of Medicine, University of Pittsburgh School of Medicine, Pittsburgh, PA, USA

Sten H. Vermund Yale School of Public Health, New Haven, CT, USA

Mary Vogler Department of Medicine, Division of Infectious Diseases, Weill Cornell Medical College, New York, NY, USA

Drenna Waldrop-Valverde Capstone College of Nursing, The University of Alabama, Tuscaloosa, AL, USA

Bruce Walker Ragon Institute of MGH, MIT and Harvard, Cambridge, MA, USA

Howard Hughes Medical Institute, Chevy Chase, MD, USA 
Robert Walton Centre for Primary Care and Public Health, Barts and the London School of Medicine and Dentistry, Queen Mary, London, UK

Huan-You Wang Department of Pathology and Division of Hematology/ Oncology, University of California, San Diego Moores Cancer Center, La Jolla, CA, USA

Christine Wanke Department of Medicine and Infectious Diseases, Tufts University School of Medicine, Boston, MA, USA

Honorine D. Ward Sackler School of Graduate Biomedical Sciences and Tufts Medical Center, Tufts University, Boston, MA, USA

Sean Wasserman Division of Infectious Diseases and HIV Medicine, Department of Medicine, University of Cape Town, Cape Town, South Africa

Andrew J. Wawrzyniak Psychiatry and Behavioral Sciences, University of Miami Miller School of Medicine, Miami, FL, USA

Wendee M. Wechsberg Global Gender Center, RTI International, Research Triangle Park, NC, USA

Health Policy and Administration, Gillings School of Global Public Health, University of North Carolina, Chapel Hill, NC, USA

Psychology Department, North Carolina State University, Raleigh, NC, USA

Psychiatry and Behavioral Sciences, Duke University School of Medicine, Durham, NC, USA

Laura Wehrmeyer Department of Health, Behavior and Society, Johns Hopkins Bloomberg School of Public Health, Baltimore, MD, USA

Louis M. Weiss Departments of Pathology and Medicine, Albert Einstein College of Medicine, Bronx, NY, USA

Oren M. Weiss Departments of Pathology and Medicine, Albert Einstein College of Medicine, Bronx, NY, USA

Departments of Medicine, Albert Einstein College of Medicine, Bronx, NY, USA

Oren S. Weiss Department of Systems and Computational Biology, Albert Einstein College of Medicine, Bronx, NY, USA

C. William Wester Vanderbilt Institute for Global Health (VIGH), Vanderbilt University School of Medicine, Nashville, TN, USA

Kenneth C. Williams Department of Biology, Boston College, Chestnut Hill, MA, USA

Cara C. Wilson Department of Medicine, Division of Infectious Diseases, University of Colorado Anschutz Medical Campus, Aurora, CO, USA

Kay Thi Win Asia Pacific Network of Sex Workers (APNSW), Yangon, Burma 
Joseph K. Wong Department of Medicine, University of California, San Francisco (UCSF) and San Francisco VA Medical Center, San Francisco, CA, USA

Glenn Wong Nuffield Department of Medicine, University of Oxford, Oxford, Oxfordshire, UK

Ida Wong-Sefidan Department of Internal Medicine, University of California, San Diego Moores Cancer Center, La Jolla, CA, USA

Cora L. Woodward Division of Biology, California Institute of Technology, Pasadena, CA, USA

Yuntao Wu Department of Molecular and Microbiology, National Center for Biodefense and Infectious Diseases, George Mason University, Manassas, VA, USA

Li Wu Department of Veterinary Biosciences, Center for Retrovirus Research, The Ohio State University, Columbus, OH, USA

Center for Microbial Interface Biology, The Ohio State University, Columbus, $\mathrm{OH}, \mathrm{USA}$

Department of Microbial Infection and Immunity, The Ohio State University, Columbus, OH, USA

Jonathan Wu Department of Molecular and Medical Pharmacology, UCLA AIDS Institute, UCLA School of Medicine, Los Angeles, CA, USA

Joyce P. Yang Department of Psychology, University of Washington, Seattle, WA, USA

Robert Yarchoan Center for Cancer Research, National Cancer Institute, Bethesda, MD, USA

Venkat R. K. Yedavalli Molecular Virology Section, Laboratory of Molecular Microbiology, National Institute of Allergy and Infectious Diseases, National Institutes of Health, Bethesda, MD, USA

Louis-Marie Yindom Nuffield Department of Medicine, NDM Research Building, University of Oxford, Oxford, UK

Sarah Yosief Department of Neurology, University of North Carolina School of Medicine, Chapel Hill, NC, USA

Steven A. Yukl Department of Medicine, University of California, San Francisco (UCSF) and San Francisco VA Medical Center, San Francisco, CA, USA

María Cecilia Zea Department of Psychology, The George Washington University, Washington, DC, USA

Nathalie C. Zeitouni Department of Dermatology, Roswell Park Cancer Institute, Buffalo, NY, USA 
Chen Zhang Vanderbilt Institute of Global Health, Vanderbilt University School of Medicine, Nashville, TN, USA

Zhi-Ming Zheng Tumor Virus RNA Biology Section, Gene Regulation and Chromosome Biology Laboratory, Center for Cancer Research, National Cancer Institute, National Institutes of Health, Frederick, MD, USA

Xiaolei Zhuang Protein Expression Laboratory, National Institute of Arthritis and Musculoskeletal and Skin Diseases, National Institutes of Health, Bethesda, MD, USA

Laboratory of Molecular Biology, National Institute of Diabetes and Digestive and Kidney Diseases, National Institutes of Health, Bethesda, MD, USA 